\title{
Caspase-3-mediated secretion of connective tissue growth factor by apoptotic endothelial cells promotes fibrosis
}

\author{
P Laplante ${ }^{1}$, I Sirois ${ }^{1}$, M-A Raymond ${ }^{1}$, V Kokta ${ }^{2}$, A Béliveau ${ }^{1}$, A Prat ${ }^{3}$, AV Pshezhetsky ${ }^{4}$ and M-J Hébert ${ }^{\star}, 1$
}

Apoptosis of endothelial cells (ECs) is an early pathogenic event in various fibrotic diseases. In this study, we evaluated whether paracrine mediators produced by apoptotic ECs play direct roles in fibrogenesis. $\mathrm{C} 3 \mathrm{H}$ mice injected subcutaneously with serumfree medium conditioned by apoptotic ECs (SSC) showed increased skin thickness and heightened protein levels of $\alpha$-smoothmuscle actin ( $\alpha \mathrm{SMA})$, vimentin and collagen I as compared with mice injected with medium conditioned by non-apoptotic ECs. Fibroblasts exposed to SSC in vitro showed cardinal features of myofibroblast differentiation with increased stress fiber formation and expression of $\alpha$ SMA. Caspase-3 silencing in ECs prevented the release of mediators favoring myofibroblast differentiation. To identify the fibrogenic factor(s) released by ECs, the protein contents of media conditioned by either apoptotic or non-apoptotic ECs were compared using SDS-PAGE-liquid chromatography (LC)-tandem mass spectrometry (MS/MS) and two-dimensional LC-MS/MS. Connective tissue growth factor (CTGF) was the only fibrogenic protein found increased in SSC. Pan-caspase inhibition with ZVAD-FMK or caspase-3 silencing in ECs confirmed that CTGF was released downstream of caspase-3 activation. The fibrogenic signaling signatures of SSC and CTGF on fibroblasts in vitro were similarly Pyk2-, Src-family kinases- and PI3K dependent, but TGF- $\beta$-independent. CTGF-immunodepleted SSC failed to induce myofibroblast differentiation in vitro and skin fibrosis in vivo. These results identify caspase-3 activation in ECs as a novel inducer of CTGF release and fibrogenesis.

Cell Death and Differentiation (2010) 17, 291-303; doi:10.1038/cdd.2009.124; published online 4 September 2009

The development of fibrosis follows a common pattern of injury and sustained repair in most tissues. ${ }^{1,2}$ In the initial phases of wound healing and chronically during fibrosis, fibroblasts accumulate at the injury site and differentiate into myofibroblasts, a contractile fibroblast type characterized by the presence of stress fibers and $\alpha$-smooth-muscle actin $(\alpha \mathrm{SMA}){ }^{2}$ Once repair is completed, the myofibroblasts undergo apoptosis and are cleared from the injury site. In fibrosis, however, the myofibroblasts develop resistance to apoptosis, preventing their clearance and leading to tissue contraction, deformation and loss of function. .,3 $^{1,3}$

Endothelial cell (EC) apoptosis is increasingly recognized as an early pathogenic event in fibrosis. A wide array of fibrogenic conditions, such as systemic sclerosis, ${ }^{4,5}$ graftversus-host disease ${ }^{6,7}$ and chronic rejection of solid allografts, ${ }^{8,9}$ has been associated with increased EC apoptosis. Pathophysiological pathways linking endothelial apoptosis to fibrogenesis are still poorly defined. Some models indicate that EC apoptosis favors the recruitment of professional phagocytes such as macrophages. ${ }^{8,10}$ Upon engulfment of apoptotic cells, the macrophages produce increased amounts of transforming growth factor- $\beta 1$ (TGF- $\beta 1$ ). ${ }^{11}$ In turn, TGF- $\beta 1$ would favor myofibroblast differentiation and resistance to apoptosis in fibroblasts/myofibroblasts. ${ }^{2}$ However, emerging evidence suggests that TGF- $\beta 1$-independent pathways also contribute to fibrogenesis. For instance, dermal fibroblasts derived from patients with diffuse scleroderma constitutively express connective tissue growth factor (CTGF), another key fibrogenic protein, in a TGF- $\beta 1$-independent manner. ${ }^{12,13}$ In addition, subcutaneous injection of CTGF into neonatal NIH Swiss mice stimulates the formation of granulation tissue and fibrosis in the absence of TGF- $\beta 1$ overproduction. ${ }^{14}$ Therefore, further characterization of TGF- $\beta 1$-independent pathways is critical to defining new targets of intervention in fibrotic conditions.

Apart from cell-cell interactions between apoptotic cells and professional phagocytes, recent results from our group have suggested that paracrine mediators produced by apoptotic ECs could play direct roles in fibrogenesis by regulating resistance to apoptosis and differentiation of fibroblasts. ${ }^{15,16}$ In the present work, we aimed to establish the fibrogenic role of this paracrine loop in vivo and to identify the paracrine mediators mediating this fibrogenic activity. We demonstrate that caspase-3 activation in apoptotic ECs

\footnotetext{
${ }^{1}$ CRCHUM (Centre de Recherche du Centre Hospitalier de l'Université de Montréal)—Hôpital Notre-Dame and Université de Montréal, Montreal, QC, Canada; ${ }^{2}$ Department of Pathology, Dermatopathology Division, Hôpital Sainte-Justine, Montreal, QC, Canada; ${ }^{3}$ Neuroimmunology Research Laboratory, Centre for Excellence in Neuromics, CHUM-Hôpital Notre-Dame and Université de Montréal, Montreal, QC, Canada and ${ }^{4}$ Research Centre, Hôpital Sainte-Justine, Montreal, QC, Canada *Corresponding author: M-J Hébert, Lab M-9208, Department of Nephrology, University of Montreal, 1560 Sherbrooke East, Montreal, QC, Canada H2L 4M1.

Tel: + 514890 8000, ext. 25393; Fax: + 514412 7661; E-mail: marie-josee.hebert.chum @ssss.gouv.qc.ca

Keywords: apoptosis; endothelial cells; myofibroblast differentiation; fibrosis

Abbreviations: N, normal medium; SS, serum-free medium; SSC, serum-free medium conditioned by apoptotic ECs; SSC-ZVAD, serum-free medium conditioned by non-apoptotic ECs; SSC-siCasp3, serum-free medium conditioned by caspase-3-silenced HUVECs; CTGF, connective tissue growth factor; LG3, C-terminal laminin G motif of perlecan; mEC, mouse EC; mSSC, mouse SSC; SYF, fibroblasts deficient in Src, Fyn and Yes

Received 12.12.08; revised 10.6.09; accepted 14.7.09; Edited by G Nunez; published online 04.9.09
} 
triggers CTGF secretion, which in turn induces myofibroblast differentiation and fibrogenesis through TGF- $\beta 1$-independent pathways.

\section{Results}

Mediators released by apoptotic ECs induce fibrosis in vivo. HUVECs exposed to serum-free medium for $4 \mathrm{~h}$ develop a significant apoptotic response in the absence of morphological features of necrosis, as evaluated by Hoechst 33342 (2'-(4-ethoxyphenyl)-5-(4-methyl-1-piperazinyl)-2.5'bi-1H-benzimidazole $(\mathrm{Ho}))$ and propidium iodide $(\mathrm{Pi})$ staining (Supplementary Figure 1). Previously we showed that serum-free medium conditioned by apoptotic ECs (SSC) induces myofibroblast differentiation in vitro. ${ }^{15}$ To verify the ability of SSC to promote fibrogenesis in vivo, mouse ECs (mECs) were isolated and cultured to generate mouse SSCs (mSSCs) (Supplementary Figure 2a). Serum starvation for $4 \mathrm{~h}$ also induced a significant apoptotic response in $\mathrm{mEC}$ (Supplementary Figure 2b). SSC conditioned for $4 \mathrm{~h}$ by apoptotic $\mathrm{mEC}$ was collected and injected subcutaneously in $\mathrm{C} 3 \mathrm{H}$ mice daily for 3 weeks. Subcutaneous injections of bleomycin and non-conditioned SS served as positive and negative controls, respectively. ${ }^{17}$

Increased skin thickness was observed in mice injected daily with mSSC as compared with mice injected with fresh SS (Figure 1a and b). The increment of skin thickness was similar to or greater than that occurring with bleomycin injections, a classical skin fibrosis model (Figure 1b). The histological changes associated with mSSC injection were characterized by dense accumulation of extracellular matrix (ECM) components and the presence of mononuclear cells in the deepest dermis layer (Figure 1a, zoom 1). Prominent disruption of the fat layer with connective tissue interposition was seen in mice treated with SSC (Figure 1a, zoom 2). These changes were comparable to those observed in bleomycin-treated mice. Western blotting of total skin protein extracts revealed heightened levels of $\alpha S M A$ and vimentin in mSSC- as compared with SS-injected mice (Figure 1c). Collagen I accumulation also was increased in mSSC- as compared with SS-treated mice (Figure 1d). Collagen I was found to be an important component of newly fibrotic lesions in both submuscular and fat layers (Figure 1d, zoom 1 and 2).

\footnotetext{
Caspase-3 activation fosters secretion of fibrogenic mediators by ECs. To demonstrate that fibrogenic mediators are released by ECs specifically in association with apoptosis, we generated media conditioned either by apoptotic or by non-apoptotic ECs (Supplementary Figure 1). For generation of serum-free medium conditioned by nonapoptotic ECs (SSC-ZVAD), an equal number of confluent ECs were either pre-incubated with the cell-permeable and irreversible pan-caspase inhibitor ZVAD-FMK $(100 \mu \mathrm{M})$ for $2 \mathrm{~h}$ or transfected with caspase-3 small interfering RNAs (siRNAs). ECs were then exposed to an equal volume of serum-free medium for $4 \mathrm{~h}$ (Supplementary Figure 1). For production of medium conditioned by apoptotic ECs, an equal number of HUVECs were pre-incubated with vehicle (DMSO) for $2 \mathrm{~h}$ or treated with control siRNAs followed by
}

serum starvation for $4 \mathrm{~h}$ (Supplementary Figure 1). Pancaspase inhibition with ZVAD-FMK or caspase-3 silencing significantly decreased the percentage of apoptotic ECs, as evaluated by Ho-Pi staining, compared with the corresponding controls in the absence of necrotic morphological features (Supplementary Figure $1 \mathrm{~b}$ and $\mathrm{c}$ ). Fibroblasts were then exposed to these conditioned media for 7 days. Fibroblasts exposed to SSC-DMSO showed heightened $\alpha \mathrm{SMA}$ levels and increased stress fiber formation, unlike fibroblasts exposed to SSC-ZVAD, which did not (Figure 2a and $c$ ). Also, serum-free medium conditioned by caspase-3silenced HUVECs (SSC-siCasp3) failed to induce myofibroblast differentiation, whereas SSC control did (Figure 2b). These data demonstrate a central role for caspase activation and more specifically activated caspase- 3 in the production of fibrogenic mediators by apoptotic ECs. As serum starvation induces a pure apoptotic response in the absence of either primary or secondary necrosis, these data also demonstrate that caspase activation triggers the release of fibrogenic mediators in the absence of abnormal cell permeability.

To further determine the importance of caspase activation in the release of fibrogenic mediators active in vivo, we compared the SSC activity generated from mECs pretreated or not with ZVAD-FMK. mSSC-ZVAD and mSSC-DMSO were generated by pre-incubating mECs with ZVAD or DMSO for $2 \mathrm{~h}$, as described above, followed by serum starvation for $4 \mathrm{~h}$. As expected from our earlier studies, serum starvation induced apoptosis in DMSO-treated mECs but not in ZVAD-treated mECs (Supplementary Figure 2b). mSSCZVAD-injected mice showed a significant reduction of skin fibrosis and thickness as compared with mSSC-DMSOinjected mice (Figure $3 a$ and $b$ ). There were no significant differences in skin thickness between mice injected with $\mathrm{mSSC}$ or those injected with mSSC-DMSO, both harboring the hallmarks of skin fibrosis (Figure $3 b$ ). Reduced $\alpha \mathrm{SMA}$ and vimentin protein levels were found in mSSC-ZVAD- as compared with mSSC-DMSO-injected mice (Figure 3c). Collagen I accumulation was also lower in mSSC-ZVADthan in mSSC-DMSO-treated mice (Figure 3a). Collectively, these results establish that caspase activation in ECs is required for the release of fibrogenic mediators active in vivo.

Characterization of the fibrogenic signaling signature induced by SSC. We showed previously that caspase-3 activation in ECs triggers the release of the C-terminal laminin G motif of perlecan (LG3). ${ }^{8,16}$ Yet, fibroblasts exposed to LG3 in vitro failed to differentiate into myofibroblasts (Supplementary Figure 3). This suggested that caspase- 3 activation supports the release of various mediators active on target cells.

To identify the key mediator(s) present in SSC and responsible for its fibrogenic activity, we adopted two parallel strategies. First, we set out to characterize the fibrogenic signaling signature induced by SSCs in fibroblasts. Second, we used a comparative proteomics approach to identify proteins with known fibrogenic activity. The fibrogenic signature of SSCs would then serve to validate that the mediator identified by comparative proteomics reproduces the fibrogenic signaling pattern induced by SSC. 
a
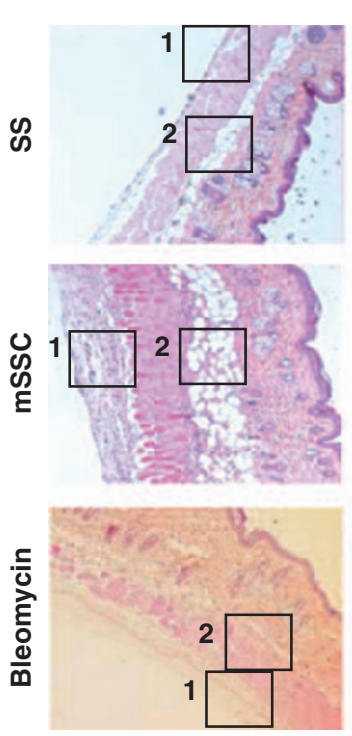

C

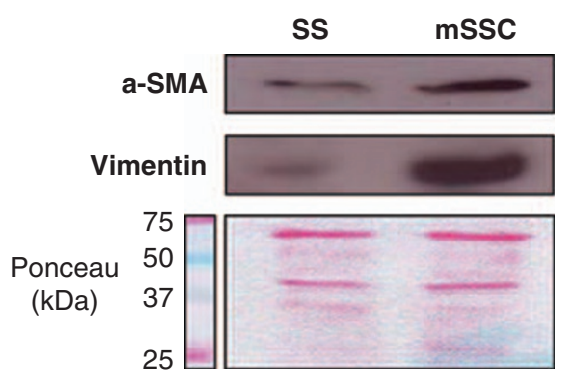

zoom 1
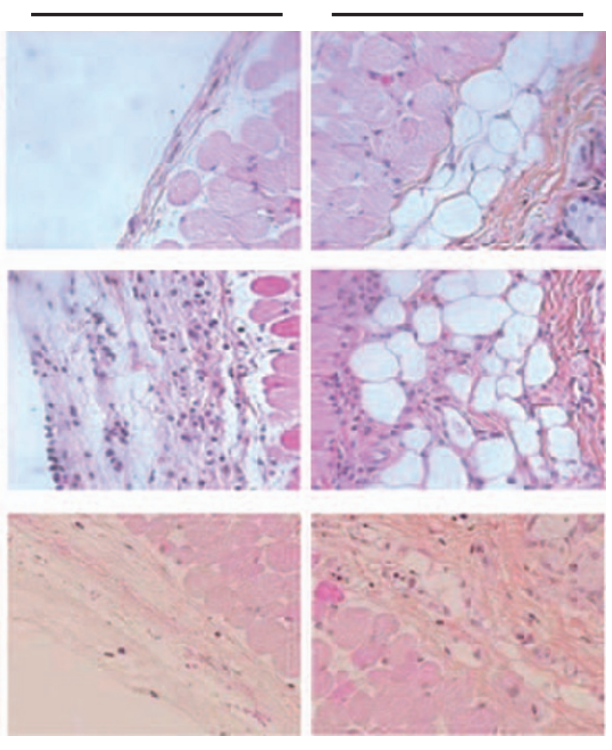

d
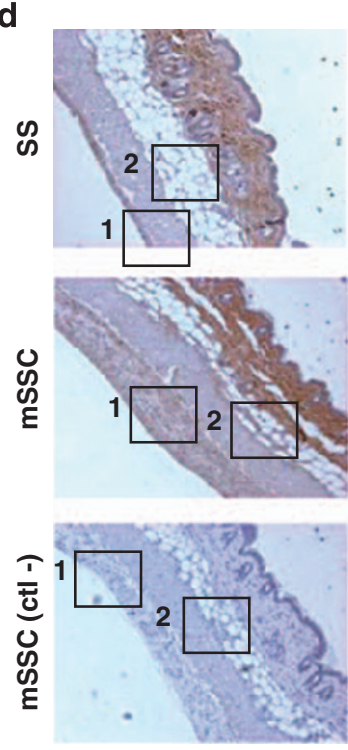

b

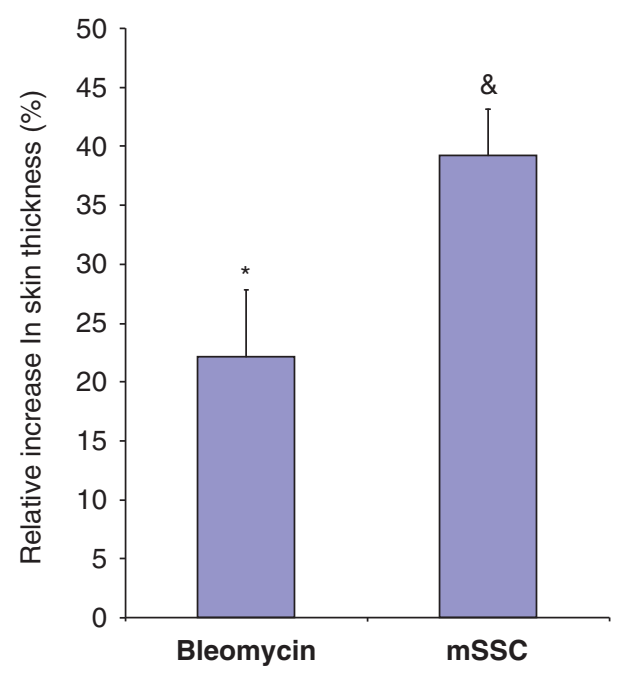

zoom 1
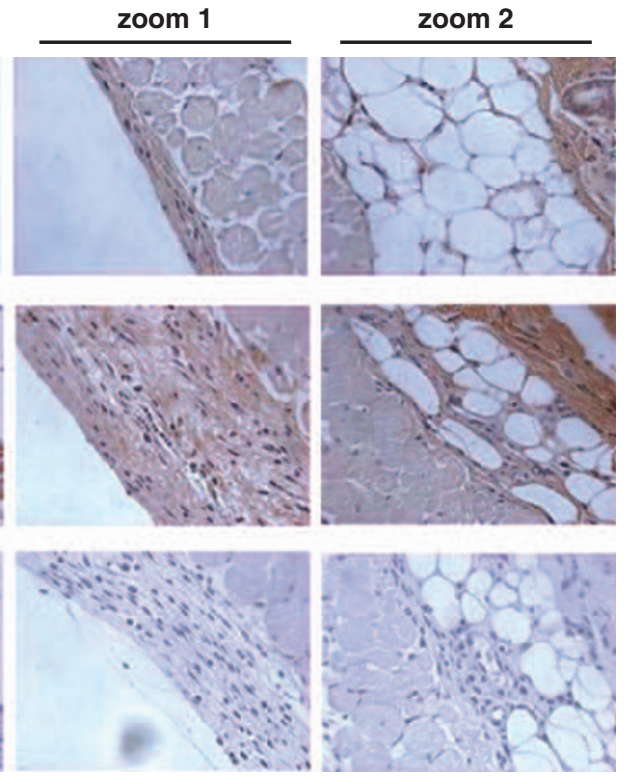

Figure 1 Apoptosis of ECs induces dermal fibrosis. (a) Histopathological evaluation of dermal fibrosis in C3H mice receiving daily subcutaneous injections of SS, $m S S C$ or bleomycin for 3 weeks. Representative HPS-stained sections (magnification $\times 100$ ); $n=8$ per group. Zoom 1 and 2 show higher magnifications of fibrotic areas within submuscular and fat layers (magnification $\times 400)$ (b) Dermal thickness was increased in mice treated with bleomycin or mSSC for 3 weeks. The results are expressed as percent increases versus the respective controls. ${ }^{*} P<0.02$ versus PBS and ${ }^{\&} P<0.04$ versus SS; $n=6$ per mouse (c) Immunoblotting analysis: Elevated $\alpha$ SMA (upper panel) and vimentin (lower panel) protein levels in the skin of mice treated with mSSC for 3 weeks as compared with mice treated with SS; $n=6$ per condition (d) Collagen I was detected immunohistochemically in tissue sections from SS- and mSSC-treated mice. Areas of collagen I deposition stained brown. The micrographs are representative of four mice per group (magnification $\times 100$ ). Zoom 1 and 2 are, respectively, higher magnifications of submuscular and fat layers demonstrating the presence of collagen I (magnification $\times 400$ ). All tissue sections were counterstained with hematoxylin, and isotype-matched IgG served as negative controls mSSC (ctl-) for collagen I

SSC induces myofibroblast differentiation through PI3Kdependent pathways, ${ }^{15}$ but upstream PI3K activators have not yet been characterized. Src-family kinases (SFKs) and focal adhesion kinase (FAK) are prime candidates as they are known PI3K activators and have been associated with fibrosis. ${ }^{18,19}$ Decreased $\alpha$ SMA protein levels and reduced stress fiber formation were found in human and mouse fibroblasts exposed to SSC in the presence of 4-amino-5-(4chlorophenyl)-7-(t-butyl)pyrazolo[3,4-d]pyrimidine (PP2), a broad inhibitor of SFK, as compared with fibroblasts exposed to SSC alone or to the inactive control, 4-amino-7-phenylpyrazol[3,4-d]pyrimidine (PP3) (Figure 4a-c). Fibroblasts isolated from $\mathrm{Src}^{-1-}, \mathrm{Fyn}^{-1-}$ (Supplementary Figure 4) and $\mathrm{Src}^{-1-} \mathrm{Fyn}^{-1-}$ double-knockout mice were tested to specifically address the role of SFK in fibrogenesis induced by SSC. $\mathrm{Src}^{-1-} \mathrm{Fyn}^{-1-}$ fibroblasts exposed to SSC for 7 days showed elevated $\alpha$ SMA protein levels as compared with those exposed to SSC alone (Figure 4c). PP2 blocked the 


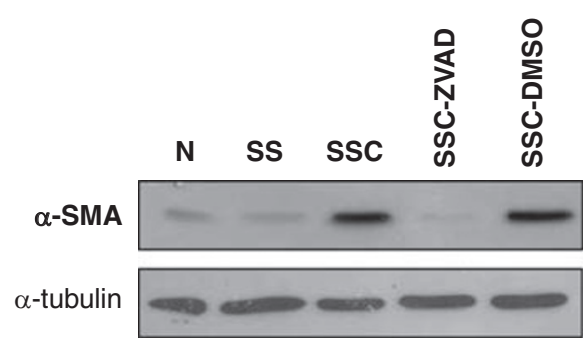

b
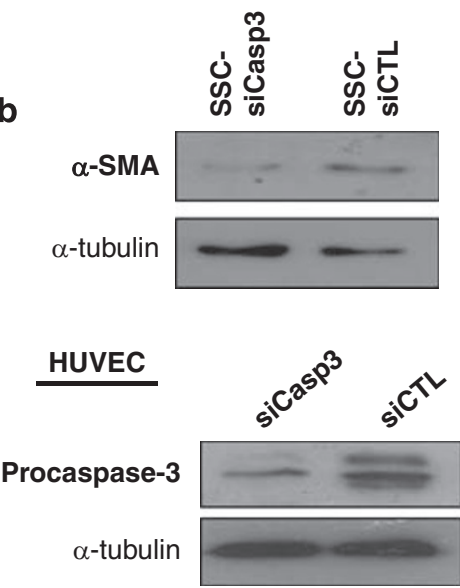

C

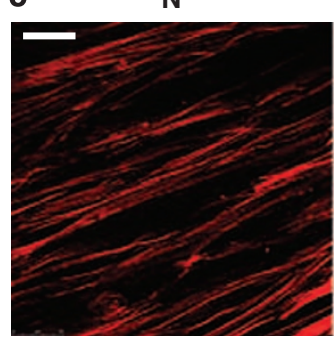

Ss

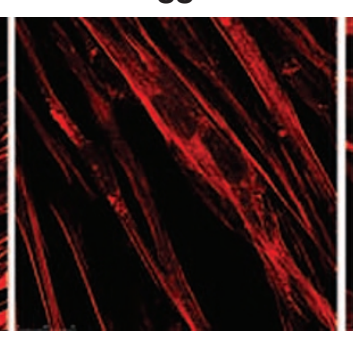

SSC

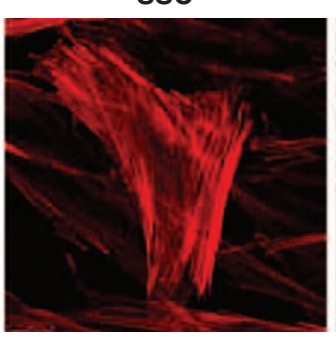

SSC-ZVAD

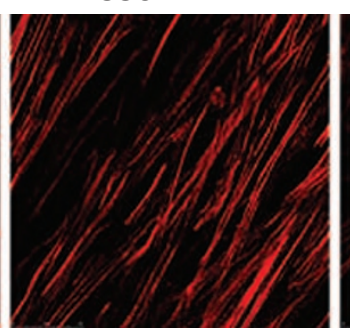

SSC-DMSO

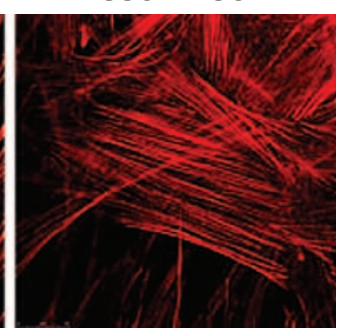

Figure 2 Caspase-3 activation in ECs is required for release of mediators that induce myofibroblast differentiation. (a) Immunoblotting analysis: Elevated $\alpha$ SMA protein levels in WI-38 fibroblasts exposed for 7 days to serum-free medium conditioned by apoptotic EC (SSC) or medium conditioned by apoptotic EC pre-incubated with DMSO (SSC-DMSO), as compared with N, SS and medium conditioned by EC in which apoptosis was inhibited with ZVAD-FMK (SSC-ZVAD). These results are representative of 3 independent experiments. (b) Immunoblotting analysis: Upper panel shows elevated $\alpha$ SMA levels in WI-38 fibroblasts exposed for 7 days to serum-free medium conditioned by apoptotic ECs pre-incubated with control siRNA (SSC-siCTL), as compared with SSC-siCasp3. Lower panel shows caspase-3 protein levels in HUVECs treated with either caspase-3 siRNAs or control siRNAs. These results are representative of three independent experiments. (c) Confocal microscopy: Rhodamine-phalloidin staining showing increased stress fiber formation in WI-38 fibroblasts exposed for 7 days to SSC and SSC-DMSO as compared with N, SS and SSC-ZVAD. The micrographs are representative of three independent experiments. Bar, $25 \mu \mathrm{m}$

upregulation of $\alpha$ SMA protein levels in $\mathrm{Src}^{-1-}, \mathrm{Fyn}^{-1-}$ and $\mathrm{Src}^{-1-} \mathrm{Fyn}^{-1-}$ as compared with SSC alone or PP3 (Figure 4c and Supplementary Figure 4). However, fibroblasts deficient in Src, Fyn and Yes (SYF) failed to upregulate $\alpha$ SMA protein levels after exposure to SSC for 7 days (Figure 4c), indicating that the coordinated activity of Src, Fyn and Yes is required to transduce the fibrogenic signals triggered by SSC.

FAK is known to interact with SFK and to play an important role in transducing fibrogenic signals. ${ }^{20}$ FAK silencing with siRNAs decreased FAK protein levels in fibroblasts for up to 7 days (Figure 4d). However, FAK-silenced fibroblasts showed clear evidence of $\alpha$ SMA induction and stress fiber formation upon exposure to SSC (Figure $4 \mathrm{~d}$ and $\mathrm{f}$ ), suggesting that FAK is dispensable for SSC-induced myofibroblast differentiation. Proline-rich tyrosine kinase 2 (Pyk2) is another member of the FAK subfamily known to interact with SFK. ${ }^{21,22}$ siRNAs for Pyk2 effectively decreased Pyk2 protein levels (Figure 4e), prevented upregulation of $\alpha \mathrm{SMA}$ protein levels and blocked stress fiber formation in fibroblasts exposed to SSC (Figure 4e and f). Collectively, these results indicate that SFK-Pyk2-PI3K is part of the fibrogenic signaling signature mediated by SSC and could be used to validate a potential fibrogenic mediator.

CTGF is released by apoptotic ECs. Two complementary proteomic approaches were adopted to characterize the fibrogenic proteins secreted by apoptotic HUVECs downstream of caspase activation: SDS-PAGE-liquid chromatography (LC)-tandem mass spectrometry (MS/MS) and two-dimensional (2D)-LC-MS/MS. ${ }^{23,24}$ To be considered of potential significance in this fibrogenic loop, the identified proteins had to meet two screening criteria: (1) human proteins identified at a relative abundance ratio of 2.5 and above in SSC-DMSO as compared with SSC-ZVAD (intensity of peptides identified in SSC-DMSO/intensity of peptides identified in SSC-ZVAD) and (2) human proteins with a known fibrogenic activity. CTGF was the only protein that met these criteria (Figure 5a). To confirm that caspase activation regulates CTGF release, equal volumes of SSCDMSO and SSC-ZVAD (human and mouse) conditioned for $4 \mathrm{~h}$ by an equal number of ECs were loaded onto a gel, and CTGF levels were compared by western blotting (Figure $5 b$ and c). Increased CTGF levels were observed in SSC-DMSO and $\mathrm{mSSC}$-DMSO as compared with their respective controls.

To further define the involvement of caspases in the secretion of CTGF, HUVECs were serum-starved in the presence of $\mathrm{N}$-benzyloxycarbonyl-Leu-Glu-His-Asp-fluoromethylketone (LEHD-FMK), N-benzyloxycarbonyl-Ile-GluThr-Asp-fluoromethylketone (IETD-FMK) or N-benzyloxycarbonyl-Asp-Glu-Val-Asp-fluoromethylketone (DEVD-FMK), which are, respectively, the inhibitors of caspase- 9 , caspase-8 and caspase-3. Both LEHD-FMK and DEVD-FMK 
a

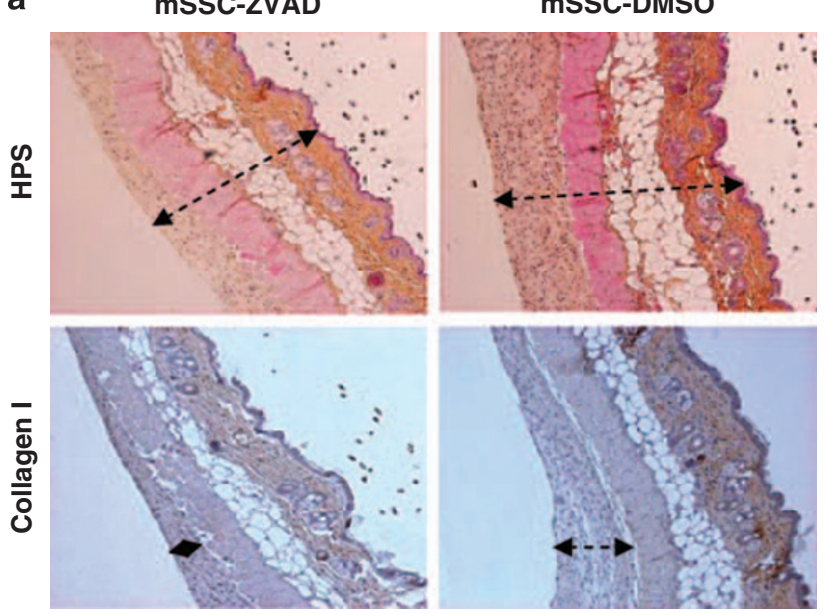

b

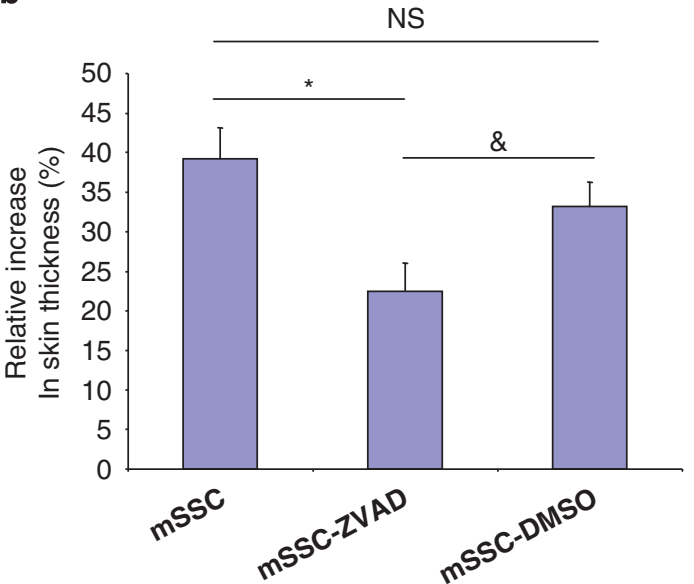

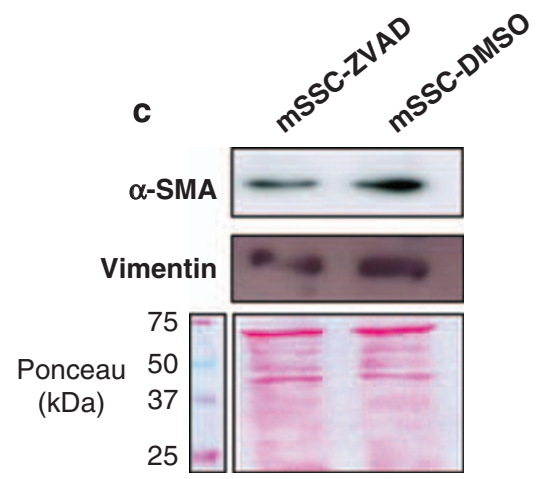

Figure 3 Caspase activation in ECs induces the release of fibrogenic mediators. (a) Upper panel: Light microscopy of HPS-stained skin sections from C3H mice injected daily with mSSC-ZVAD or mSSC-DMSO (magnification $\times 100$ ). Fibrosis increased in mSSC-DMSO-injected mice; $n=4$ per group. Lower panel: Collagen I was detected immunohistochemically in tissue sections from mSSC-ZVAD- and mSSC-DMSO-treated mice. The micrographs (magnification $\times 100$ ) are representative of four mice per group. All tissue sections were counterstained with hematoxylin. Fibrotic areas, which were larger in mice treated with mSSC-DMSO, were positive for collagen I immunostaining. (b) Decreased skin thickness in mice treated with mSSC-ZVAD for 3 weeks. The results are expressed as percent increases versus SS. ${ }^{*} P<0.004$ versus mSSC and ${ }^{\&} P<0.04$ versus mSSC-DMSO; $n=4$ per group. (c) Immunoblotting analysis: Skin protein extracts from mice treated with mSSC-DMSO for 3 weeks showed heightened $\alpha$ SMA (left panel) and vimentin (right panel) levels compared with the respective mSSC-ZVAD controls; $n=6$ per condition

inhibited apoptosis of serum-starved HUVECs and attenuated the release of CTGF. IETD-FMK, which failed to decrease apoptosis in serum-starved HUVECs, also failed to inhibit CTGF export (Figure 5d). These results suggest that the effector caspase-3 plays a central role in the regulation of CTGF export. To further evaluate this possibility, HUVECs were transfected with caspase-3 siRNAs or control before serum starvation. Caspase-3 silencing in serum-starved HUVECs inhibited the development of apoptosis (as evaluated by Ho-Pi staining; Supplementary Figure 1c) and significantly attenuated the secretion of CTGF, further substantiating the pivotal role of caspase- 3 activation in CTGF export (Figure 5e).

To further define the kinetics of CTGF secretion in association with development of apoptosis, we evaluated the time courses of EC apoptosis, caspase-3 activation, PARP cleavage and CTGF export. The kinetics of caspase-3 activation (Figure 6a), PARP cleavage (Figure 6a) and CTGF export (Figure $6 \mathrm{~b}$ ) were closely matched and preceded the development of nuclear changes characteristic of apoptosis (Figure 6a). There was no evidence of either primary or secondary necrosis in HUVECs serum starved for up to $4 \mathrm{~h}$ as evaluated by Ho-Pi staining and PARP cleavage (Figure 6a). These results suggest that necrosis does not contribute to the secretion of CTGF in this system. Finally, we also considered the kinetics of CTGF release as compared with the potential release of other intracellular constituents or caspase-3 substrate. No PARP signals were found in medium conditioned by apoptotic endothelial cells for $4 \mathrm{~h}$ (data not shown), whereas, at the same time point, extracellular CTGF levels were already increased (Figure 6c). In addition, intracellular proteins, such as Grp94, an endoplasmic reticulum protein, or tubulin, were not released by apoptotic serum-starved ECs (Figure 6c). These results show that the extracellular release of CTGF does not result from unspecific protein leakage.

We then evaluated whether the release of CTGF during apoptosis depends on the pro-apoptotic stimulus. HUVECs were exposed to mitomycin C (MMC) or tumor necrosis factor$\alpha(\mathrm{TNF} \alpha)$, which are, respectively, activators of the intrinsic and extrinsic apoptotic pathways, for $4 \mathrm{~h}$ in the normal medium (N). Both mediators increased PARP cleavage in ECs and also enhanced the secretion of CTGF (Figure 6d). Further, lower concentrations of both mediators, which failed to induce apoptosis in HUVECs, as evaluated by PARP cleavage, also 
failed to increase the secretion of CTGF. Collectively these results show a key role of the effector caspase-3 in fostering CTGF export independently of the initiating stimulus.

Considering the relationship between CTGF and heightened TGF- $\beta$ 1-dependent signaling and the involvement of the latter in fibrogenesis, we sought to explore whether
TGF- $\beta 1$ was involved in the phenotype induced by SSC on fibroblasts. It is noteworthy that TGF- $\beta$ isoforms were not found to be increased in SSC-DMSO as compared with SSCZVAD, as determined by the two proteomics approaches. Added to this, co-incubation of fibroblasts with neutralizing pan-TGF- $\beta$ antibodies failed to inhibit myofibroblast a

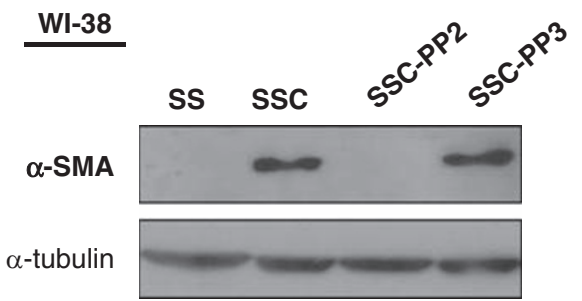

b

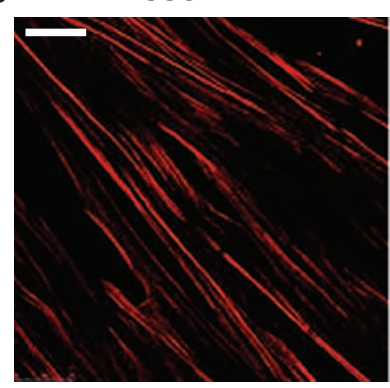

SSC-PP3

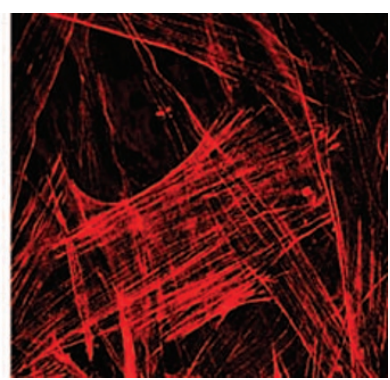

c
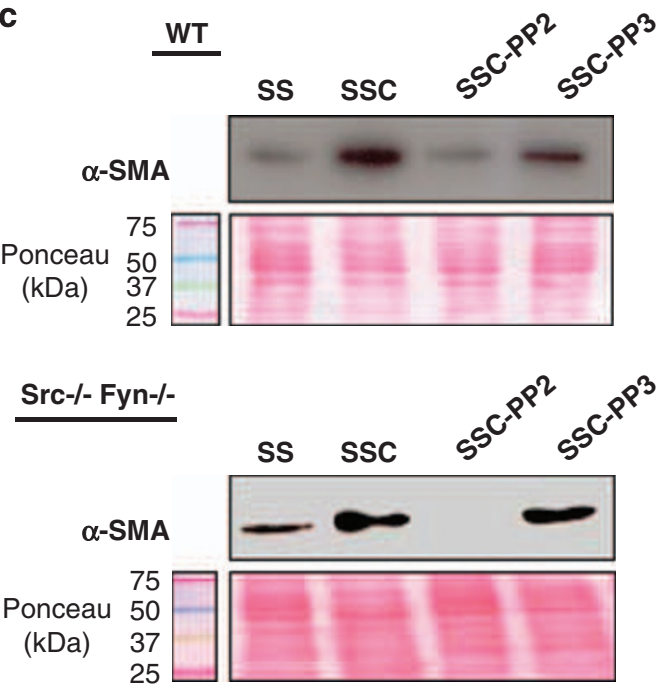

d

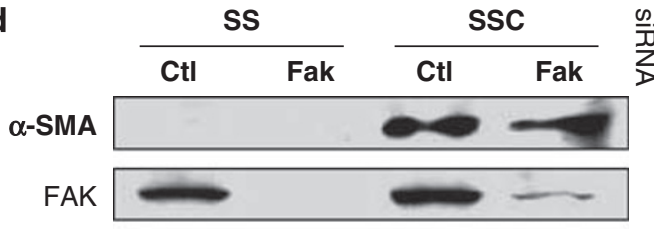

$\alpha$-tubulin

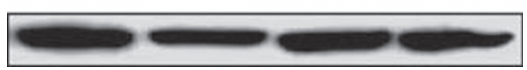

e
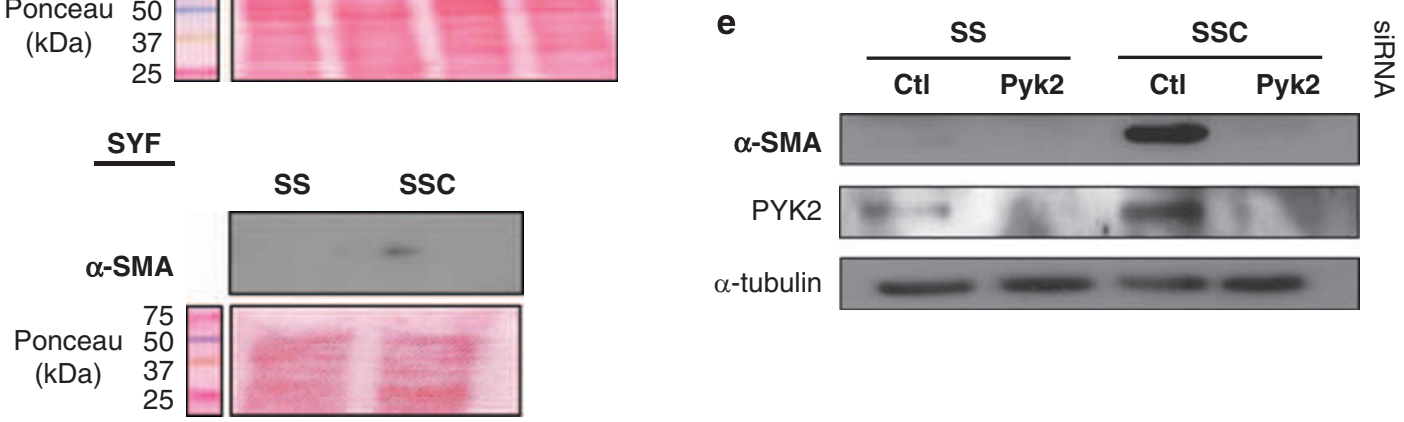

$\alpha-S M A$

PYK2

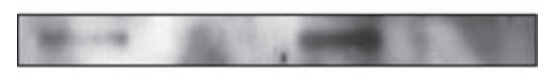

$\alpha$-tubulin
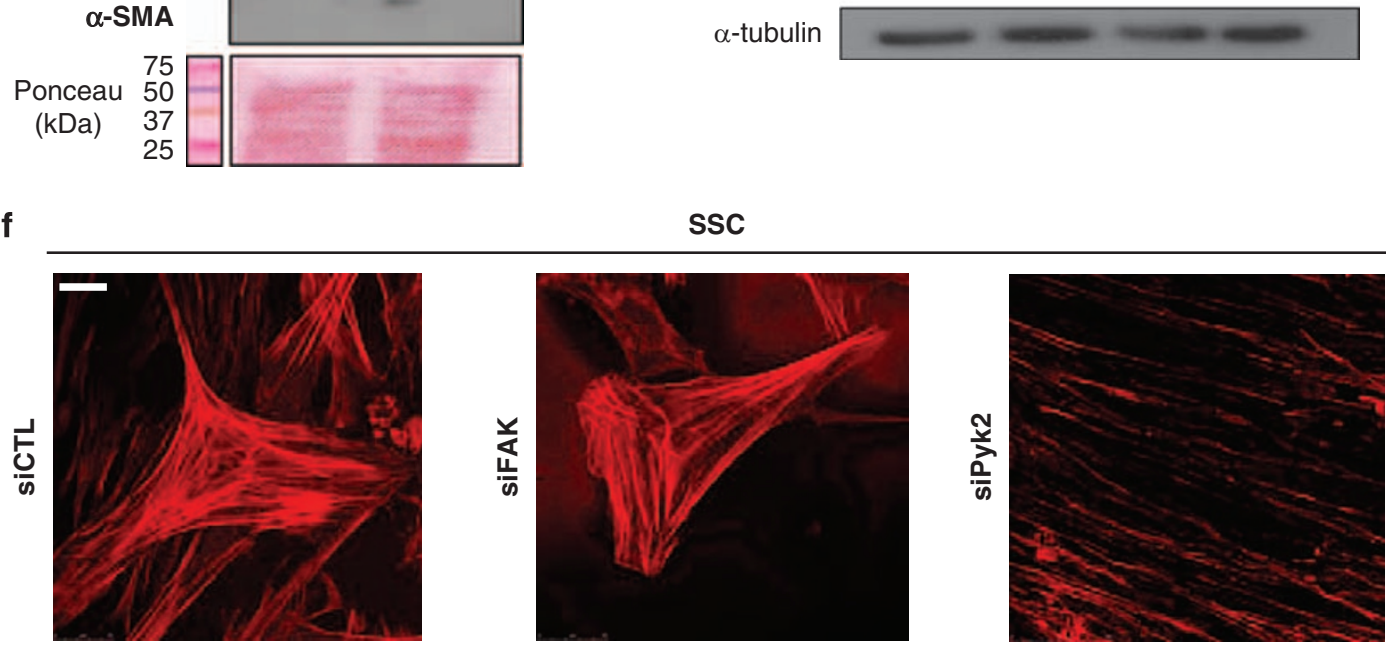
a

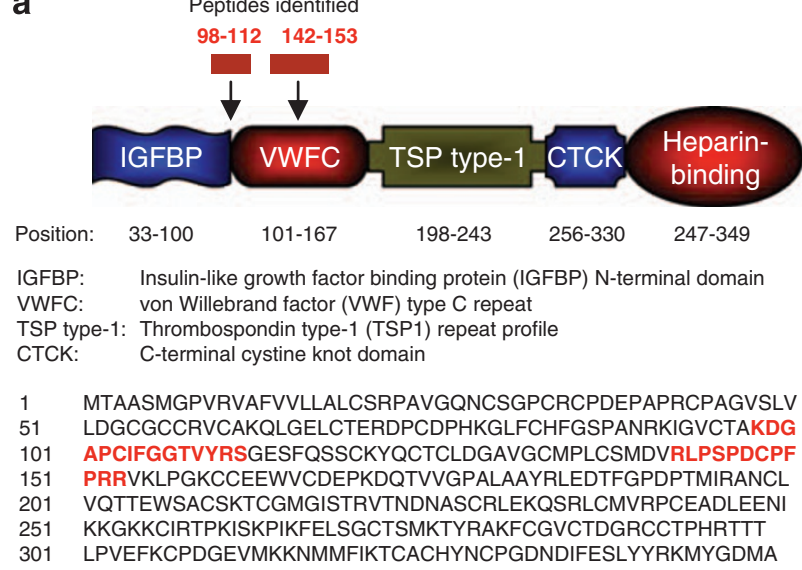

b

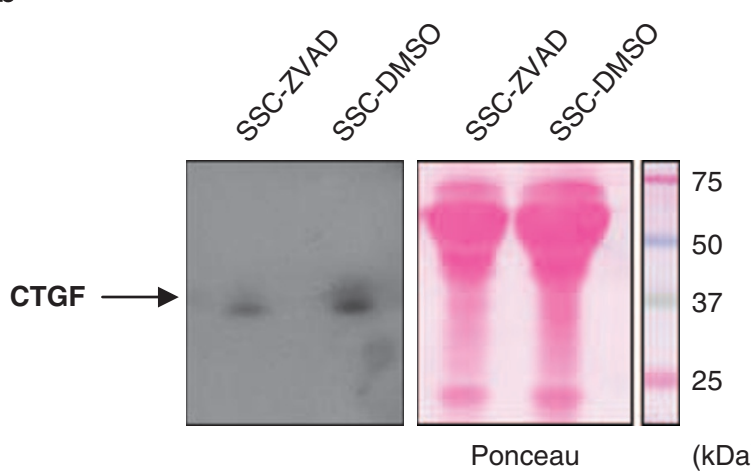

d

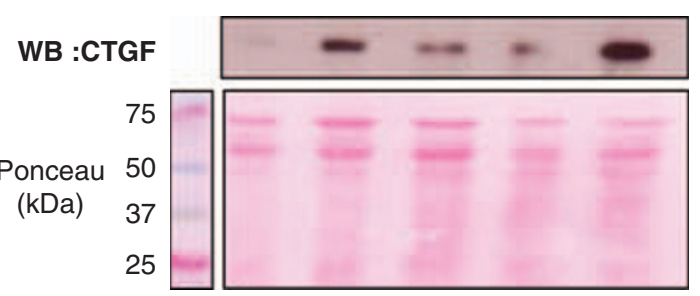

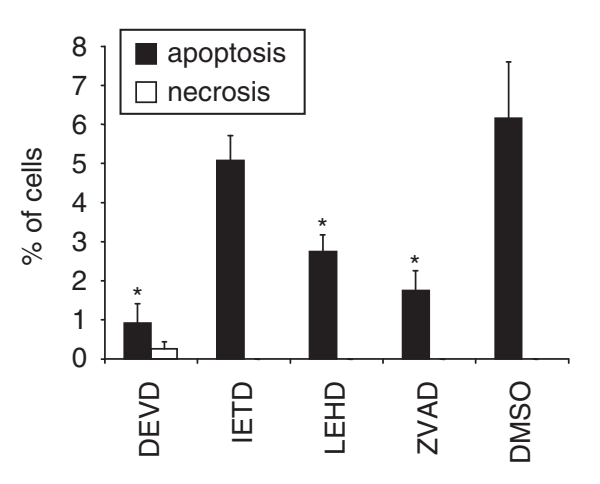

Da) e

C
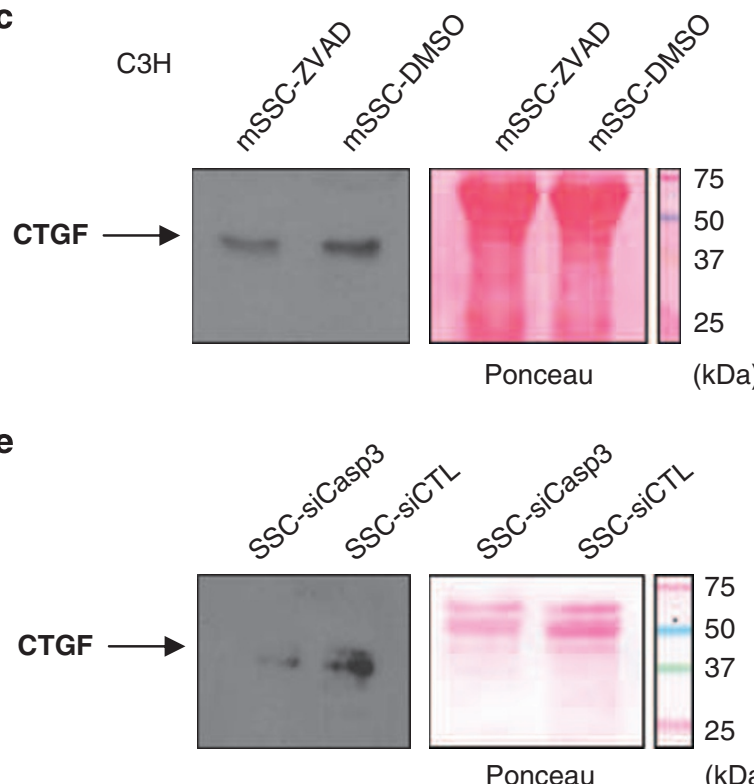

Figure 5 Caspase-3 activation triggers the release of active CTGF by apoptotic EC. (a) A schematic representation of CTGF and corresponding peptides identified by SDS-PAGE-LC-MS/MS. Sequences identified in SSC-DMSO are in red. (b, c) Immunoblotting analysis: SSC-ZVAD and SSC-DMSO, respectively generated from human and C3H ECs, were precipitated by TCA followed by Western blotting against CTGF. CTGF protein levels were increased in SSC-DMSO as compared with an equal volume of SSC-ZVAD. These results are representative of three independent experiments. (d) Percentage of apoptotic and necrotic cells (as evaluated by Ho-Pi staining) in HUVECS exposed for $4 \mathrm{~h}$ to SS with $100 \mu \mathrm{M}$ of Z-DEVD-FMK, Z-IETD-FMK, Z-LEHD-FMK, Z-VAD-FMK or control (DMSO). ${ }^{*} P<0,02$ versus DMSO, representative of six experiments. Immunoblotting analysis: Decreased levels of CTGF in medium recovered from the above conditions (DEVD, LEHD and ZVAD) as compared with DMSO or IETD. Ponceau S Red staining of the entire membrane is shown as a loading control. These results are representative of four independent experiments. (e) Immunoblotting analysis: SSC-siCTL and SSC-siCasp3 were precipitated by TCA followed by Western blotting against CTGF. CTGF protein levels were augmented in SSC-siCTL as compared with an equal volume of SSC-siCasp3. The results are representative of four independent experiments

Figure 4 Importance of SFK and Pyk2 in myofibroblast differentiation induced by SSC. (a) Immunoblotting analysis: Decreased $\alpha$ SMA protein levels in WI-38 fibroblasts exposed to SSC in the presence of PP2 $(5 \mu \mathrm{M})$ for 7 days, as compared with inactive control PP3. These results are representative of six independent experiments. (b) Confocal microscopy: Rhodamine-phalloidin staining showing increased stress fiber formation in WI-38 fibroblasts exposed for 7 days to SSC-PP3 as compared with SSCPP2. The micrographs are representative of three independent experiments. Bar, $25 \mu \mathrm{m}$. (c) Immunoblotting analysis: Decreased $\alpha$ SMA protein levels in wild type (WT) and $\mathrm{Src}^{-1-} \mathrm{Fyn}^{-1-}$ mouse fibroblasts exposed to SSC in the presence of PP2 $(5 \mu \mathrm{M})$ for 7 days, as compared with the controls (PP3). SYF cells exposed for 7 days to SSC do not show elevated $\alpha$ SMA protein levels compared with SS. Ponceau S Red staining was included as a loading control. The results are representative of three independent experiments. (d) Immunoblotting analysis: Fibroblasts transfected with control siRNA or FAK siRNA and exposed to SSC for 7 days showed similar $\alpha$ SMA protein levels, whereas FAK levels were decreased in FAK-silenced fibroblasts. The results are representative of five independent experiments. (e) Immunoblotting analysis: Fibroblasts transfected with Pyk2 siRNA and exposed to SSC for 7 days showed decreased $\alpha$ SMA and Pyk2 protein levels as compared with fibroblasts transfected with control siRNA. These results are representative of three independent experiments. (f) Confocal microscopy: Rhodamine-phalloidin staining of stress fibers in WI-38 fibroblasts transfected with control siRNA or siRNA against FAK or Pyk2 and exposed to SSC for 7 days. Stress fiber formation is inhibited in Pyk2-silenced fibroblasts. The micrographs are representative of two independent experiments. Bar, $25 \mu \mathrm{m}$ 
a Intracellular
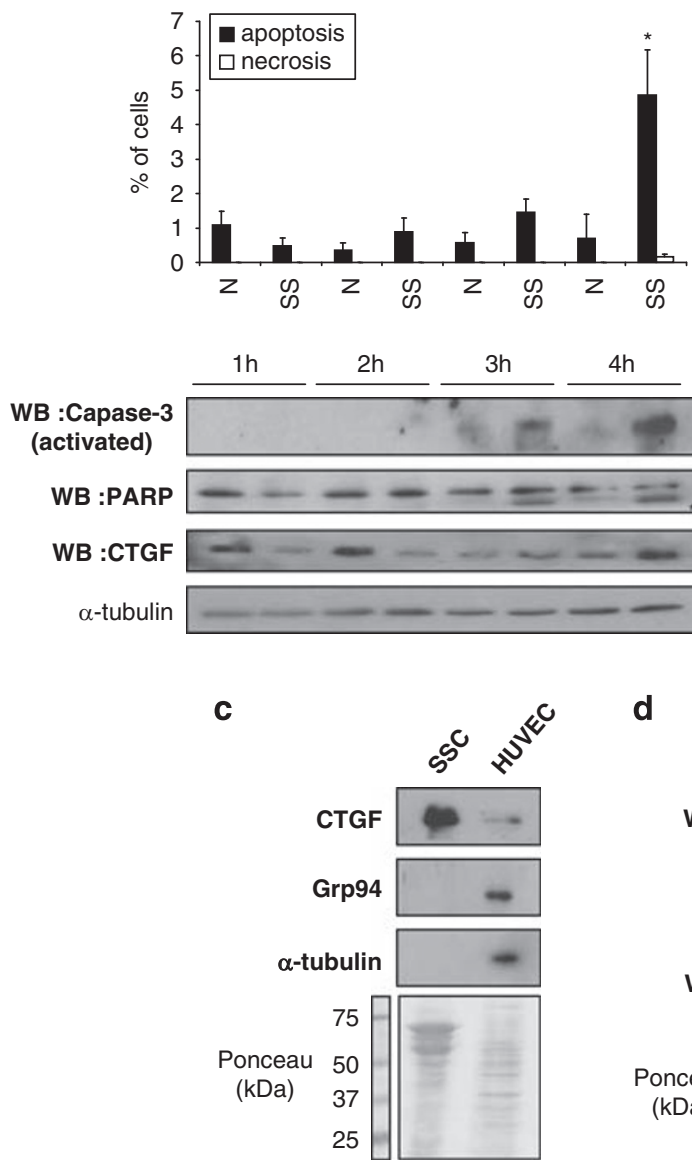

b

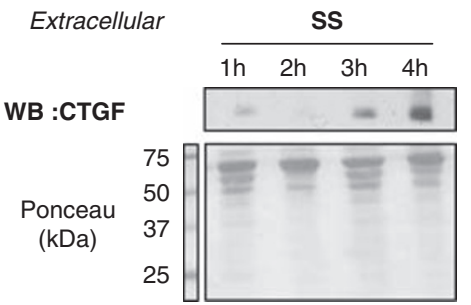

Figure 6 Time-dependent release of CTGF by apoptotic EC. (a) Percentage of apoptotic and necrotic cells (evaluated by Ho-Pi staining) in ECs exposed for $1 \mathrm{~h}, 2 \mathrm{~h}, 3 \mathrm{~h}$ and $4 \mathrm{~h}$ to $\mathrm{N}$ or SS. ${ }^{*} P<0,05$ versus $\mathrm{N}$, representative of five experiments. Immunoblotting analysis: Increased levels of activated caspase-3 and PARP cleavage in ECs exposed to SS for $3 \mathrm{~h}$ or $4 \mathrm{~h}$ as compared with ECs exposed to N. Intracellular CTGF protein levels also increases over time in serum-starved ECs. These results are representative of three independent experiments; ( ${ }^{*}$ represents the $89-\mathrm{kDa}$ cleaved fragment of PARP characteristic of apoptosis). (b) Immunoblotting analysis: Serum-free media conditioned by ECs for 1 and up to $4 \mathrm{~h}$. The secretion of CTGF increases over time in -starved HUVECs. Ponceau S Red staining of the entire membrane is shown as a loading control. These results are representative of three independent experiments. (c) Immunoblotting analysis: CTGF is present in cell extracts obtained from ECs and in medium conditioned by apoptotic ECs serum starved for $4 \mathrm{~h}$, whereas Grp-94 and $\alpha$-tubulin are only detected in cell extracts. These results are representative of three independent experiments. (d) Immunoblotting analysis: Increased intracellular PARP cleavage by apoptotic ECs (MMC $100 \mu \mathrm{g} / \mathrm{ml}$ and TNF $\alpha 1 \mu \mathrm{g} / \mathrm{ml}$ ) as compared with nonapoptotic ECs (MMC $10 \mu \mathrm{g} / \mathrm{ml}$ and TNF $\alpha 100 \mathrm{ng} / \mathrm{ml}$ ). Extracellular CTGF levels in corresponding conditioned media. Ponceau S Red staining of the entire membrane is shown as a loading control. These results are representative of three independent experiments ( ${ }^{*}$ represents the $89-\mathrm{kDa}$ cleaved fragment of PARP characteristic of apoptosis)

differentiation of fibroblasts exposed to SSC or CTGF, whereas myofibroblast differentiation induced by recombinant TGF- $\beta 1$ was significantly reduced (Figure $7 \mathrm{a}$ ). These data suggest that the fibrogenic paracrine pathways activated by EC apoptosis are largely TGF- $\beta$ independent and point to CTGF as a central mediator.

CTGF induces a fibrogenic signaling signature characteristic of SSC. We then evaluated whether the fibrogenic signaling signature evoked by CTGF in fibroblasts was similar to that observed with SSC. Human WI-38 fibroblasts exposed for 7 days to SS supplemented with recombinant CTGF $(10 \mathrm{ng} / \mathrm{ml})$ showed augmented $\alpha \mathrm{SMA}$ levels (Figure 7b). Co-incubation with PP2 and LY2940002, inhibitors of SFK and PI3K, respectively, blocked CTGFdependent $\alpha$ SMA upregulation in human and mouse fibroblasts (Figure 7b and Supplementary Figure 5). In agreement with our previous work, ${ }^{15,16}$ heightened Akt phosphorylation was found in fibroblasts exposed to SSC (Figure 7c). CTGF also increased Akt phosphorylation in fibroblasts, which was inhibited in the presence of PP2 (Figure 7c). CTGF also enhanced stress fiber formation in fibroblasts, which was inhibited by Pyk2 silencing (Figure 7d). Finally, SYF fibroblasts failed to upregulate aSMA upon exposure to CTGF for 7 days (Figure 7e). Collectively, our findings demonstrate similar signaling signatures for CTGF and SSC.

To further demonstrate the key role of CTGF in this paracrine fibrogenic loop, SSC was immunodepleted of CTGF (Figure 8a). Fibroblasts exposed for 7 days to immunodepleted SSC showed decreased aSMA levels as compared with fibroblasts exposed to either SSC alone or 


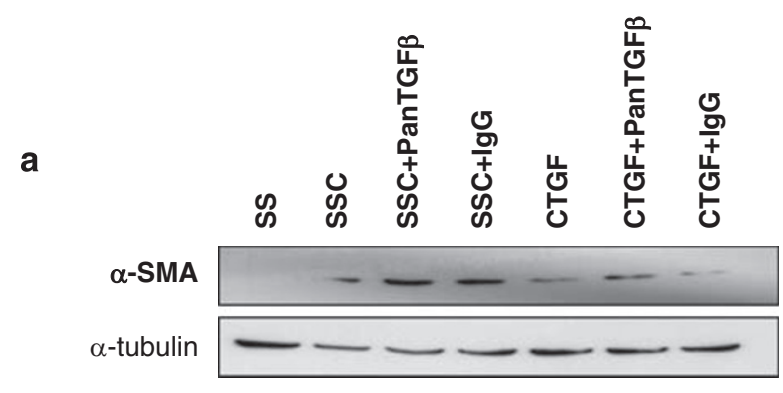

b

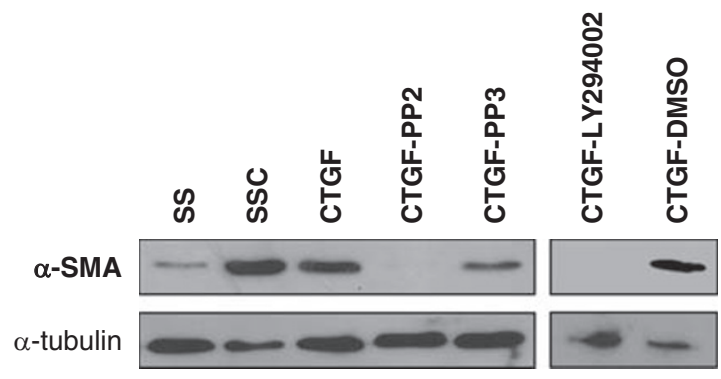

d

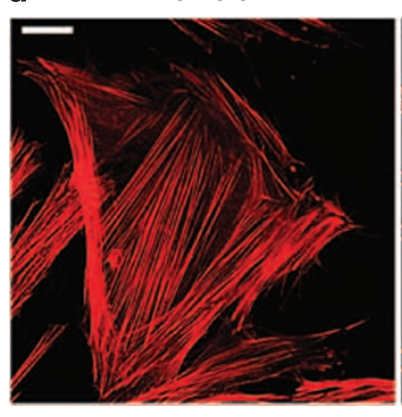

vehicle

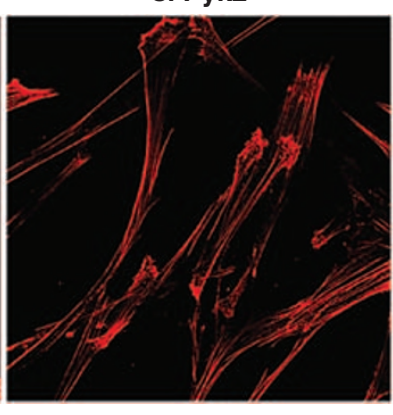

CTGF

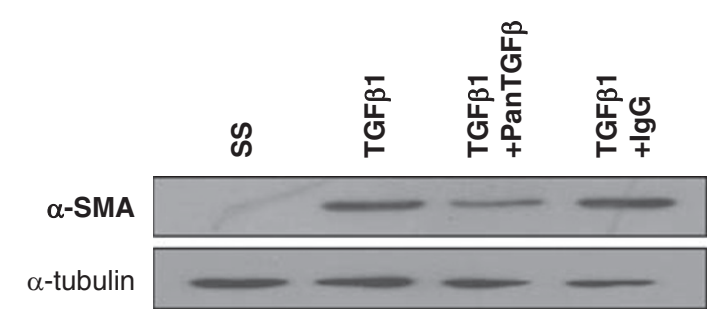

c

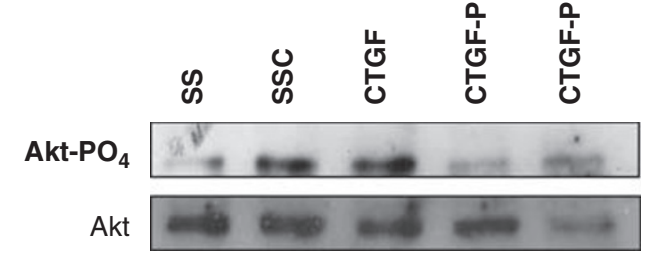

si CTL

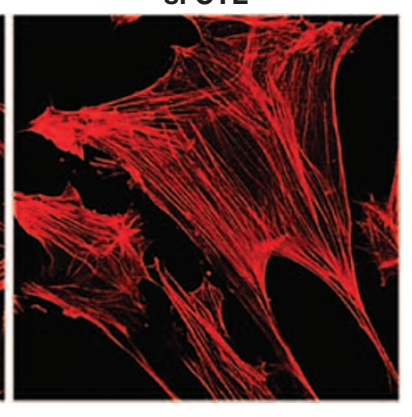

e SYF

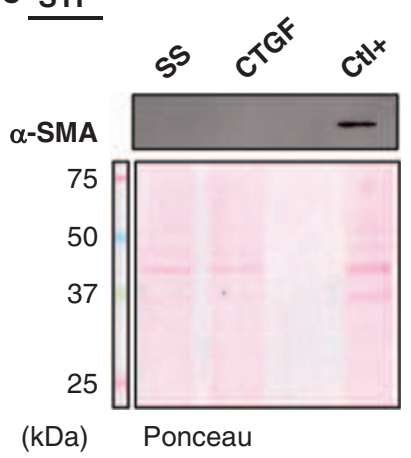

Figure 7 CTGF recapitulates the signaling signature of SSC. (a) Left panel: Immunoblotting analysis of fibroblasts exposed for 7 days to SSC or CTGF (10 ng/ml), either alone, with a pan-TGF- $\beta$-blocking antibody $(5 \mu \mathrm{g} / \mathrm{ml})$ or with isotype-matched lgG, showed elevated $\alpha$ SMA protein levels as compared with fibroblasts exposed to SS. Right panel: Immunoblotting analysis of fibroblasts exposed for 7 days to TGF- $\beta 1(2 \mathrm{ng} / \mathrm{ml})$ with a pan-TGF- $\beta$-blocking antibody $(5 \mu \mathrm{g} / \mathrm{ml})$ manifested decreased $\alpha$ SMA protein levels as compared with fibroblasts exposed to TGF- $\beta 1$ alone or with isotype-matched IgG. These results are representative of four independent experiments. (b) Immunoblotting analysis: Elevated $\alpha$ SMA protein levels in fibroblasts exposed for 7 days to SS supplemented with CTGF (10 ng/ml) alone or concomitantly with PP3 or DMSO. The results are representative of three independent experiments. (c) Immunoblotting analysis: Decreased Akt phosphorylation in fibroblasts exposed for $4 \mathrm{~h}$ to serumfree medium supplemented with CTGF and PP2 $(5 \mu \mathrm{M})$. These results are representative of three independent experiments. (d) Confocal microscopy: Rhodamine-phalloidin staining in fibroblasts non-transfected (vehicle) or transfected with Pyk2 siRNA or control siRNA and exposed for 7 days to CTGF. Stress fiber formation was decreased in fibroblasts transfected with Pyk2 siRNA. The micrographs are representative of two independent experiments. Bar, $25 \mu \mathrm{m}$. (e) Immunoblotting analysis: SYF mouse fibroblasts exposed for 7 days to CTGF do not show elevated $\alpha$ SMA protein levels as compared with SS. Ponceau S Red staining of the entire membrane was included as a loading control. WI-38 fibroblasts exposed for 7 days to SSC are positive controls $(\mathrm{Ctl}+)$ for $\alpha$ SMA expression. These results are representative of three independent experiments

SSC treated with an isotype-matched inactive antibody (Figure 8a). Altogether, these observations indicate that CTGF is a major fibrogenic mediator released by apoptotic ECs.

CTGF released by apoptotic ECs induces skin fibrosis. To evaluate the role of CTGF as an initiator of skin fibrosis in vivo, we compared the fibrogenic activities of SSCs immunodepleted of CTGF or control. Mice were injected subcutaneously daily for 3 weeks with $300 \mu \mathrm{l}$ of CTGF-immunodepleted mSSCs (mIP-CTGF) or mSSCs treated with an isotype-matched non-depleting control (mIP-Ctl). Skin fibrosis and thickness were significantly reduced in mice injected with mIP-CTGF as compared with mIP-Ctl (Figure $8 \mathrm{~b}$ and $\mathrm{c}$ ). $\alpha \mathrm{SMA}$ and vimentin protein levels were also reduced in mIP-CTGF-treated mice as compared with mIP-Ctl-treated mice (Figure $8 \mathrm{~d}$ ). Collagen I accumulation was also reduced in IP-CTGF-treated mice as compared with IP-Ctl-treated mice (Figure $8 \mathrm{~b}$ ). Taken together, our results demonstrate that apoptotic ECs release increased CTGF levels, which, in turn, plays a central role in triggering fibrogenic pathways in vivo.

\section{Discussion}

Fibrosis is associated with sustained accumulation of myofibroblasts responsible for the production of ECM components, 
a
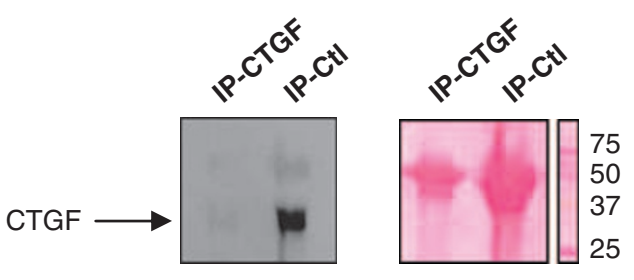

Ponceau (kDa)

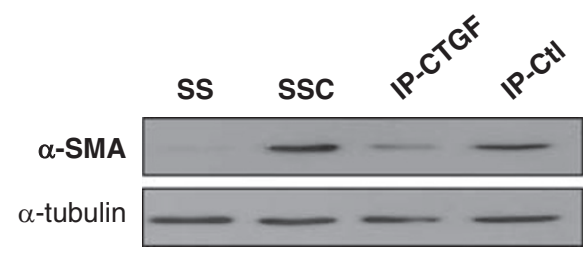

b

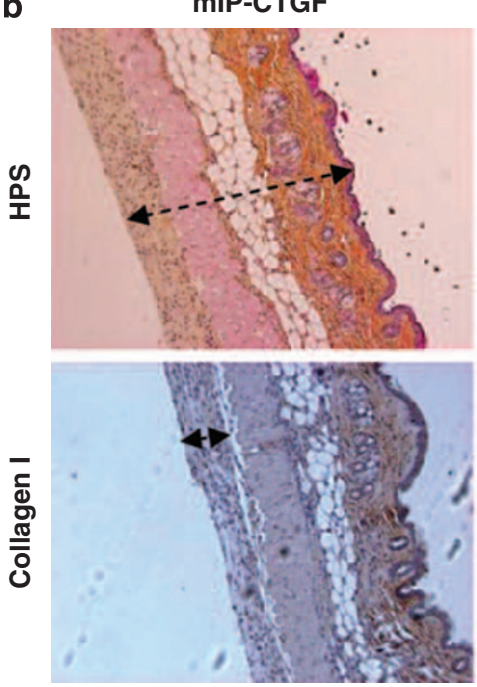

mIP-Ctl

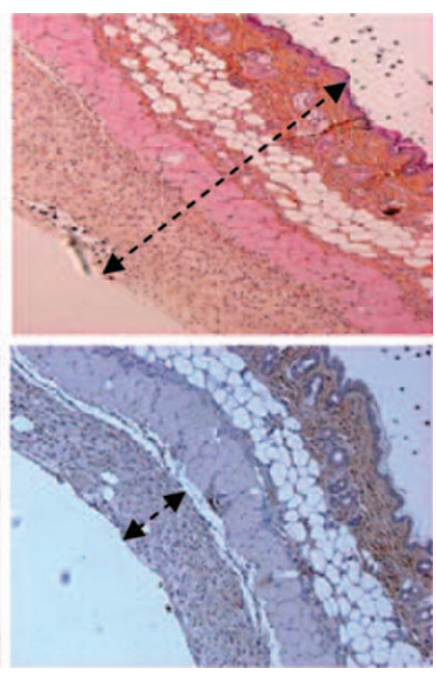

C

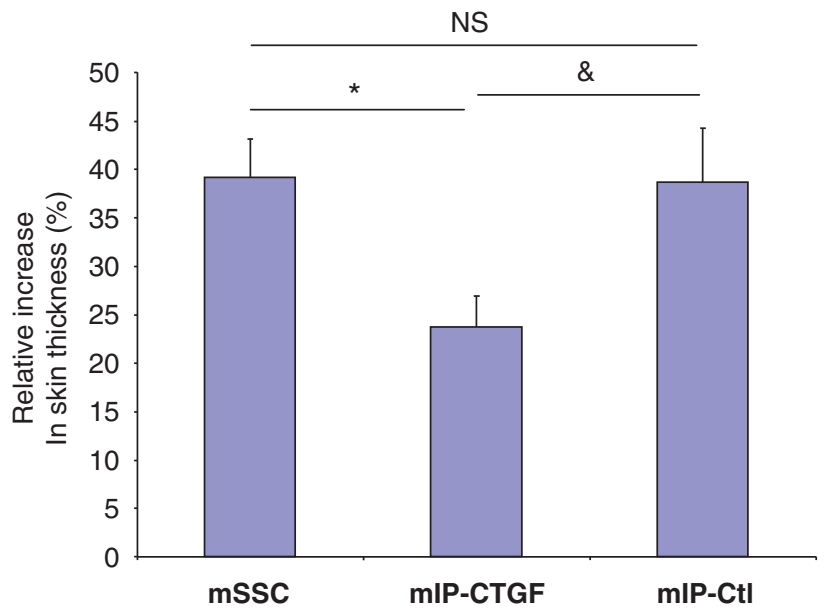

d
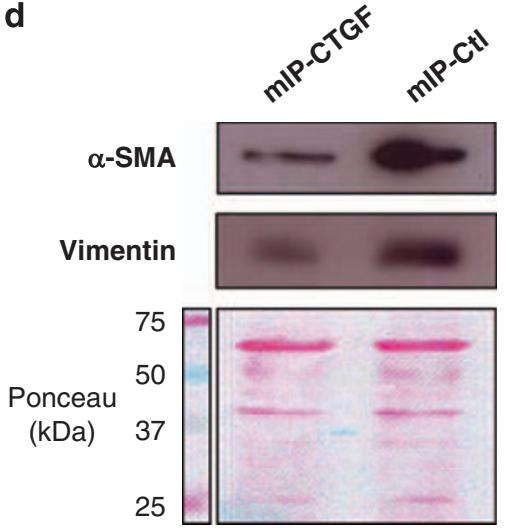

Figure 8 CTGF is a key fibrogenic mediator released by apoptotic EC. (a) Upper panel shows immunoblotting analysis of CTGF protein levels in SSC immunodepleted of CTGF (IP-CTGF) or SSC exposed to isotype-matched immunoglobulin (IP-Ctl). Ponceau S Red staining of the entire membrane is shown as loading control. Lower panel shows immunoblotting analysis of fibroblasts exposed for 7 days to IP-CTGF showing decreased $\alpha$ SMA protein levels as compared with fibroblasts exposed to SSC or IP-Ctl. These results are representative of three independent experiments. (b) Upper panel: Light microscopy of HPS-stained skin sections from C3H mice injected daily with mIP-CTGF or its control (mIP-Ctl) (magnification $\times 100$ ). Fibrosis was increased in mIP-Ctl-injected mice; $n=6$ per group. Lower panel: Collagen I was detected immunohistochemically in tissue sections from mIP-CTGF- and mIP-Ctl-treated mice. The micrographs (magnification $\times 100$ ) are representative of four mice per group. All tissue sections were counterstained with hematoxylin. Fibrotic areas, which were larger in mice treated with mIP-Ctl, were positive for collagen I immunostaining. (c) Decreased skin thickness in mice treated with mIP-CTGF for 3 weeks. The results are expressed as percent increases versus SS. ${ }^{\star} P<0.004$ versus mSSC and ${ }^{\&} P<0.03$ versus mIP-Ctl; $n=6$ per group. (d) Immunoblotting analysis: Skin protein extracts from mice treated with mIP-Ctl for 3 weeks showed elevated $\alpha$ SMA (upper panel) and vimentin (lower panel) levels as compared with mice treated with mIP-CTGF; $n=6$ per condition

tissue contraction and loss of function. Inappropriate and sustained resistance to apoptosis in myofibroblasts prevents their clearance and fuels fibrogenic reactions. Intriguingly, and as opposed to myofibroblasts, increased EC apoptosis has been reported to play a primary pathogenic role in various fibrotic diseases such as chronic rejection of solid organ transplants, graft-versus-host disease and systemic sclerosis. $^{4,5,25}$

The fibrogenic pathways elicited by EC apoptosis are still poorly defined. Here, we aimed at evaluating whether paracrine mediators produced by apoptotic ECs could activate fibrogenic pathways in vivo. Subcutaneous injection of recombinant proteins such as TGF- $\beta$ or CTGF in rodents has played a key role in characterizing the fibrogenic activity of these mediators. ${ }^{26}$ In addition, subcutaneous injection of bleomycin in mice is a classical model of fibrosis. ${ }^{17}$ Hence, to address the fibrogenic activity of the paracrine component of apoptosis, EC apoptosis was induced ex vivo, and the mediators produced by apoptotic ECs were collected and injected in vivo. This approach showed that paracrine mediators produced by apoptotic ECs induce the key characteristics of fibrosis in vivo, such as increased skin thickness and heightened expression of $\alpha \mathrm{SMA}$, vimentin and collagen I. The fibrogenic response evoked by subcutaneous SSC injection in mice was similar to that obtained with bleomycin. 
The critical role of caspase activation in controlling the secretion of fibrogenic mediators was confirmed by showing that the medium conditioned by caspase-inhibited ECs elicited significantly less fibrosis when injected in vivo than the medium conditioned by apoptotic ECs. Further, the medium conditioned by caspase-inhibited or caspase-3silenced ECs failed to induce myofibroblast differentiation in vitro, unlike the medium conditioned by apoptotic ECs. These results show a pivotal role for effector caspase-3 activation in the induction of paracrine fibrogenic pathways.

LG3 is produced downstream of caspase-3 activation in apoptotic ECs. ${ }^{8}$ Yet, in this system, LG3 failed to favor myofibroblast differentiation, indicating that caspase-3 activation in ECs promotes the release of various factors with specialized functions; some mediators, such as LG3, elicit resistance to apoptosis in fibroblasts, whereas others regulate myofibroblast differentiation.

We used a comparative proteomics approach to identify additional mediators released by apoptotic ECs and with known fibrogenic activity. However, identification of a specific protein by MS/MS does not necessarily translate into functional activity, as the identified peptides could represent degraded fragments or, inversely, unprocessed precursor proteins, both without biological activity. Hence, we also sought to characterize the fibrogenic signaling signature of SSC with the aim of focusing on fibrogenic mediator(s) evoking the same signaling pattern. Unexpectedly, only one protein with known fibrogenic activity, CTGF, was identified by MS/MS, and its increased protein levels in the medium conditioned by apoptotic ECs were confirmed by Western blotting. Biochemical caspase inhibition and transfection with siRNAs showed that increased CTGF release by apoptotic ECs occurs through caspase-3-dependent pathways. Caspase-3 activation prompted a rapid export process, as CTGF release occurred concomitantly with PARP cleavage, preceded nuclear apoptotic changes and developed in the absence of secondary (or primary) necrosis. Finally, CTGF release was independent of the apoptotic stimulus, further supporting the contention that CTGF export is specifically regulated at the level of the execution phase of the apoptotic process.

CTGF, a 37-kDa cysteine-rich peptide, belongs to the CCN family of matricellular proteins. ${ }^{27}$ Through its $\mathrm{N}$-terminal domain, it has been shown to mediate myofibroblast differentiation and collagen synthesis, whereas its C-terminal domains have been implicated in the regulation of proliferation and adhesion. ${ }^{28}$ Consistent with CTGF's functional activity in our system, sequences identified by MS/MS analysis corresponded to the N-terminal domains. No TGF- $\beta$ isoforms were found to be increased in SSC; yet, CTGF is known to potentiate interactions between low levels of TGF- $\beta 1$ and its cognate receptor. ${ }^{29}$ Hence, we considered the possibility of TGF- $\beta 1$-dependent signaling in this fibrogenic loop, even in the absence of elevated amounts of TGF- $\beta 1$. Surprisingly, blockade of TGF- $\beta$ signaling with a pan-TGF- $\beta$-neutralizing antibody did not prevent myofibroblast differentiation induced by either SSC or recombinant CTGF. These results are in keeping with our previous work ${ }^{15}$ showing low TGF- $\beta 1$ levels in SSC, and indicate a predominant role of TGF- $\beta 1$ independent pathways in our system.
To validate the importance of CTGF in this novel paracrine loop, we first turned to the fibrogenic signaling signature induced in fibroblasts in response to SSC. We had shown, in our previous work, that SSC elicited myofibroblast differentiation through PI3K-dependent pathways. ${ }^{15}$ However, the upstream signaling pathways regulating PI3K activation in our system were still uncharacterized. SFK and FAK are both considered as pathways implicated in myofibroblast differentiation, fibrosis and PI3K activation. ${ }^{18,19}$ Our experiments with biochemical inhibitors and gene-deficient fibroblasts confirmed a key function of Src, Fyn and Yes SFK in the transduction of fibrogenic signals by SSC. Surprisingly, FAK silencing did not affect myofibroblast differentiation as $\alpha$ SMA and stress fiber formation were still induced in response to SSC. However, Pyk2, which is approximately $60 \%$ identical to FAK in its central catalytic domains and shares approximately $40 \%$ identity in both the $\mathrm{N}$ - and C-terminal domains, ${ }^{30}$ was identified as a novel fibrogenic signaling effector as its silencing largely blocked the fibrogenic signals induced by SSC. Collectively, these results characterized the SFK-Pyk2PI3K pathways as important signaling components activated by SSC. Recombinant CTGF reproduced the same signaling signature in fibroblasts, and inhibition of these signaling pathways blocked CTGF-dependent myofibroblast differentiation in vitro. Finally, we also showed that immunodepleting the SSCs of CTGF blocked myofibroblast differentiation in vitro and prevented the development of fibrosis in vivo, providing compelling evidence that CTGF is pivotal in the fibrogenic paracrine loop activated by endothelial apoptosis.

CTGF is constitutively overexpressed in a number of fibrotic diseases, the most severe being systemic sclerosis. ${ }^{31}$ Elevated CTGF protein levels, reported in the serum, ${ }^{32}$ broncho-alveolar lavage ${ }^{33}$ and dermal interstitial fluid ${ }^{34}$ in systemic sclerosis patients, are an indicator of disease extent and severity. A polymorphism in the CTGF promoter region, increasing CTGF expression levels, has recently been associated with systemic sclerosis. ${ }^{35}$ CTGF has also been identified as a marker of fibrosis in chronic renal and heart allograft rejection, disease states that are also associated with sustained endothelial injury. ${ }^{36,37}$ Blockade of CTGF expression by systemic injection of siRNAs was recently shown to prevent fibrosis in a model of chronic renal allograft rejection. ${ }^{38}$ Hence, CTGF is increasingly recognized as a key fibrotic mediator in a number of severe fibrotic diseases, but the various pathways that regulate its expression and secretion are only beginning to be unraveled.

In summary, our study provides novel insights into the mediators and pathways linking EC apoptosis to fibrogenesis. Caspase-3 activation in ECs leads to the release of CTGF, which, in turn, favors myofibroblast differentiation and fibrogenesis in vivo. Myofibroblast differentiation induced by CTGF occurs largely through TGF- $\beta$-independent pathways that rely on SFK, Pyk2 and PI3K activation. These results open new areas of intervention for controlling fibrosis in situations of chronic endothelial damage and apoptosis.

\section{Materials and Methods}

Cell lines. HUVECs, obtained from Clonetics (San Diego, CA, USA) and grown in EC basal medium (Clonetics), were used between passages 2-4. WI-38 human fibroblasts from normal embryonic lung tissue were purchased from the American 
Type Culture Collection (ATCC, Rockville, MD, USA), grown in fibroblast basal medium (Cambrex, Walkersville, MD, USA) supplemented with $10 \%$ inactivated fetal bovine serum (FBS; Medicorp, Montreal, QC, Canada) and used between passages 2-17.

Fibroblasts genetically deficient in $\mathrm{Src}\left(\mathrm{Src}^{-1-}\right)$ and/or Fyn $\left(\mathrm{Fyn}^{-1-}\right)$ were isolated from mouse embryo fibroblasts homozygous for disruption of the Src and/or Fyn genes and immortalized with large $\mathrm{T}$ antigen. ${ }^{39}$ These cells were kindly provided by Jun-Ichi Abe (Center for Cardiovascular Research, University of Rochester, Rochester, NY, USA). SYF mouse fibroblasts were procured from the ATCC and cultured in Dulbecco's Modified Eagle's Medium (Wisent, Saint-Bruno, QC, Canada) supplemented with $10 \%$ inactivated FBS.

ECs were isolated from the aorta of $\mathrm{C} 3 \mathrm{H}$ mice, as described previously..$^{40}$ They were identified by immunostaining for platelet EC adhesion molecule-1 (CD31) with a goat polyclonal antibody directed against mouse CD31 (Santa Cruz Biotechnology, Santa Cruz, CA, USA). SSC, SSC-ZVAD and SSC-DMSO were generated from $\mathrm{C} 3 \mathrm{H}$ ECs, as described for HUVECs. ${ }^{8,15,16}$ In brief, an equal number of ECs were exposed to the vehicle (DMSO) or ZVAD-FMK $(100 \mu \mathrm{M})$ for $2 \mathrm{~h}$, washed, kept in SS for $4 \mathrm{~h}$ and harvested. For all experiments, equal volumes of media conditioned by an equal number of cells (apoptotic or not) were compared.

\section{Characterization of the fibrogenic proteins produced by} apoptotic ECs. We used two comparative proteomic approaches to identify the secretome released by apoptotic ECs: 2D-LC-MS/MS and SDS-PAGE-LC-MS/ MS. Serum-free media $\left(0.16 \mathrm{ml} / \mathrm{cm}^{2}\right)$ conditioned by apoptotic (SSC-DMSO) and non-apoptotic ECs pre-incubated with ZVAD-FMK (SSC-ZVAD) were centrifuged sequentially at $20000 \times g$ and $50000 \times g$ to eliminate cell debris and apoptotic blebs, and depletion was confirmed by flow cytometry (FACScan equipped with CellFit software; Beckton Dickinson, Franklin Lakes, NJ, USA) using FL1 and FL2 channels. For 2D-LC-MS/MS analysis, conditioned media were fractionated by HPLC, each fraction concentrated, solubilized and digested overnight with trypsin Promega (Madison, WI, USA), sequencing grade, estimated trypsin/protein ratio $1: 100)$. The tryptic peptides were analyzed by LC-MS/MS as previously described. ${ }^{24}$ SDS-PAGE-LC-MS/MS analysis was performed essentially as described. ${ }^{24}$ In all, $125-\mu \mathrm{g}$ weight of total protein from each sample was resolved by SDS-PAGE on a $15 \%$ acrylamide gel. The gel was stained with Coomassie blue. Each lane was cut into 20 slices and treated with trypsin (Promega). Tryptic peptides were extracted, followed by LC-MS/MS analysis (Supplementary data).

Immunoblotting. Proteins were extracted, separated by electrophoresis, transferred to nitrocellulose membranes and probed, as described previously. ${ }^{15}$ For comparison of protein levels in conditioned media, $1 \mathrm{ml}$ of each medium was precipitated with TCA 9:1 for $1 \mathrm{~h}$ on ice, centrifuged at 14000 r.p.m., washed with cold acetone and solubilized in Laemmli sample buffer, followed by western blotting against CTGF. The antibodies for western blotting were Grp-94 (Abcam Inc., Cambridge, MA, USA); anti- $\alpha$ SMA and anti-vimentin (Sigma, St. Louis, MO, USA); anti-collagen I (Biodesign International, Saco, ME, USA); anti-Pyk2 (BD Biosciences, Franklin Lakes, NJ, USA); and anti-FAK, anti-CTGF (Santa Cruz Biotechnology) and anti-PARP, anti-cleaved caspase-3 $\left(\right.$ Asp $\left.^{175}\right)$, anti-phospho-Akt $\left(\mathrm{Ser}^{473}\right.$ ) and anti-Akt (Cell Signaling Technology Inc., Beverly, MA, USA).

The membranes were stained with Ponceau S Red or, alternatively, after initial probing, they were stripped and reprobed with anti- $\alpha$-tubulin monoclonal antibody (Oncogene, Boston, MA, USA). Ponceau S Red was considered as a loading control for all experiments involving conditioned media, as a means for comparing their entire protein contents. ${ }^{8}$

Confocal microscopy for stress fiber characterization. Cells were grown on 35-mm glass-bottomed microwell dishes (MatTek Corporation, Ashland, MA, USA), rinsed with PBS and fixed with $2 \%$ formaldehyde. They were washed three times in PBS before permeabilization and after each subsequent step. Permeabilization was performed with $50 \mathrm{nM} \mathrm{NH}_{4} \mathrm{Cl}$ and $0.3 \%$ Triton X-100 in PBS for 15 min. Microwell dishes were blocked with PBS/BSA $3 \%$ for 30 min and incubated with phalloidin-tetramethylrhodamine (Sigma) for $60 \mathrm{~min}$ at room temperature. The cells were then visualized at room temperature under a Leica SP5 confocal microscope (emission detector $570-620 \mathrm{~nm}$ ) and analyzed using Leica LAS AF software.

RNA interference. WI-38 fibroblasts were plated in six-well plates or $35-\mathrm{mm}$ glass-bottomed microwell dishes at 100000 cells per well. After $20 \mathrm{~h}$ they were transfected with double-stranded RNA-DNA hybrids at a final concentration of
$200 \mathrm{~nm}$ annealed oligonucleotides and Oligofectamine (Invitrogen, Carlsbad, CA, USA). After $45 \mathrm{~h}$ of transfection, the cells were kept under experimental conditions for 7 days, followed by evaluation of myofibroblast differentiation. FAK oligonucleotides were procured from Dharmacon Research (Lafayette, CO, USA) and used as reported previously. ${ }^{16}$ Pre-designed oligonucleotides for Pyk2 (ONTARGETplus SMARTpool) were also obtained from Dharmacon Research.

HUVECs were grown in 6- and 24-well plates until $75 \%$ confluence per well. They were transfected with pre-designed oligonucleotides for caspase-3 (ON-TARGETplus SMARTpool) at a final concentration of $100 \mathrm{~nm}$ and Oligofectamine. After $16 \mathrm{~h}$, the cells were placed in $\mathrm{N}$ for $72 \mathrm{~h}$. They were then exposed to serum-free media for $4 \mathrm{~h}$, followed by evaluation of apoptosis and procaspase-3 protein levels, or production of conditioned media. ${ }^{8,15}$

Screening for apoptosis by fluorescence microscopy. Fluorescence microscopy of unfixed/unpermeabilized adherent cells stained with Ho and PI was performed as described in our previous work. ${ }^{15,16}$ The percentages of normal, apoptotic and necrotic cells adherent to the dishes were estimated by an investigator blinded to the experimental conditions.

Immunodepletion and TCA precipitation. A $10-\mathrm{ml}$ volume ${ }^{10}$ of SSC was incubated with $40 \mu \mathrm{g}$ of anti-CTGF antibody (or isotype-matched immunoglobulin $\mathrm{G}(\mathrm{IgG})$ as negative control) for $6 \mathrm{~h}$ at $4^{\circ} \mathrm{C}$ with gentle shaking. A $250-\mu$ l volume of protein A/G (Santa Cruz Biotechnology) was added, followed by overnight incubation at $4^{\circ} \mathrm{C}$ with gentle shaking. Then the media were collected, centrifuged at 14000 r.p.m. for $10 \mathrm{~min}$ to remove protein $\mathrm{A} / \mathrm{G}$ complexes and sterilized with a $0.2-\mu \mathrm{m}$ filter. TCA precipitation was performed as described above.

Murine model of skin fibrosis. Female pathogen-free, 6-week-old $\mathrm{C} 3 \mathrm{H}$ mice (Charles River Laboratories Inc., Wilmington, MA, USA) were maintained on food and water ad libitum. The protocol was approved by the Animal Care Committee of the Centre hospitalier de l'Université de Montréal (CHUM).

A $300-\mu$ l volume of conditioned media was injected subcutaneously into a single location on the shaved back of the mice daily for 3 weeks with a 27-gauge needle. Bleomycin injections $(1 \mathrm{mg} / \mathrm{ml})$ daily for 3 weeks, as described by others, ${ }^{17}$ served as positive controls. The mice ( $n=8$ per group) were killed on day 21 .

Tissue preparation and immunohistochemistry. The mice were killed 1 day after the final injection, and skin from the injection site was removed and cut into two pieces: one was fixed in 10\% formalin solution and embedded in paraffin; the other was snap-frozen in liquid nitrogen and stored immediately at $-80^{\circ} \mathrm{C}$. Sections $(3 \mu \mathrm{m})$ were cut from the embedded pieces of skin, mounted on slides and stained with hematoxylin-phloxine-saffron (HPS). Dermal thickness was measured under a light microscope by an investigator blinded to the experimental conditions in three randomly selected fields (average of three measurements per field) for each mouse. Immunohistochemical analysis of collagen I (1:50 for $2 \mathrm{~h}$ at room temperature) was performed using an $\mathrm{ABC}$ anti-rabbit staining kit, according to the manufacturer's instructions (Santa Cruz Biotechnology). Negative controls were prepared by replacing the primary antibody with isotype-matched rabbit $\lg G$ (R\&D Systems, Minneapolis, MN, USA).

Snap-frozen skin pieces were processed for isolation of proteins with TRIzol as per the manufacturer's instructions (Invitrogen), with a few modifications. Briefly, the chopped skin pieces were immersed in $1.0 \mathrm{ml}$ of ice-cold TRIzol reagent and homogenized for 2 min with a PowerGen-125 tissue grinder from Fisher Scientific (Ottawa, ON, Canada). After phase separation, the air-dried protein pellet was dissolved in $1 \%$ SDS solution and heated at $50^{\circ} \mathrm{C}$ for $5 \mathrm{~min}$ to improve protein solubility. The protein concentration of each sample was measured according to the BCA method. ${ }^{15}$

Reagents. PP2 and PP3 were purchased from Calbiochem (San Diego, CA, USA). Recombinant human CTGF and recombinant human TGF- $\beta 1$ were bought from Cell Sciences (Canton, MA, USA) and R\&D Systems, respectively. LG3 was produced as described previously. ${ }^{16}$ Caspase inhibitors (Z-DEVD-FMK, Z-IETD-FMK, Z-LEHD-FMK, and N-benzyloxycarbonyl-Val-Ala-Asp-fluoromethylketone (Z-VAD-FMK)) and pan-TGF- $\beta 1$-blocking antibody were obtained from R\&D Systems. All other reagents were from Sigma Chemicals (Oakville, ON, Canada). 
Statistical analysis. The results are expressed as means \pm S.E.M. The data were analyzed by Student's $t$-test or ANOVA, as appropriate. $P<0.05$ was considered significant for all tests.

Acknowledgements. This work was supported by research grants from the Kidney Foundation of Canada (to $\mathrm{MJH}$ ) and the Canadian Institutes of Health Research (CIHR) to MJH (MOP-89869) and AVP (MOP-66980). MJH is the holder of the Shire Chair in Nephrology, Transplantation and Renal Regeneration of the Université de Montréal. PL is the recipient of a training award from Fonds de la Recherche en Santé du Québec (FRSQ). IS is the recipient of a training fellowship from the ClHR. We thank the J.-L.Lévesque Foundation for their renewed support, as well as Mr. Romain Cayrol and Mr. Nicolas Parent for their help with confocal and light microscopy.

1. Hinz B. Formation and function of the myofibroblast during tissue repair. J Invest Dermatol 2007; 127: 526-537.

2. Tomasek JJ, Gabbiani G, Hinz B, Chaponnier C, Brown RA. Myofibroblasts and mechanoregulation of connective tissue remodelling. Nat Rev Mol Cell Bio 2002; 3: 349-363.

3. Horowitz JC, Rogers DS, Sharma V, Vittal R, White ES, Cui Z et al. Combinatorial activation of FAK and AKT by transforming growth factor-beta1 confers an anoikis-resistant phenotype to myofibroblasts. Cell Signal 2007; 19: 761-771.

4. Jun JB, Kuechle M, Harlan JM, Elkon KB. Fibroblast and endothelial apoptosis in systemic sclerosis. Curr Opin Rheumatol 2003; 15: 756-760.

5. Sgonc R, Gruschwitz MS, Boeck G, Sepp N, Gruber J, Wick G. Endothelial cell apoptosis in systemic sclerosis is induced by antibody-dependent cell-mediated cytotoxicity via CD95. Arthritis Rheum 2000; 43: 2550-2562.

6. Deschaumes C, Verneuil L, Ertault-Daneshpouy M, Adle-Biassette H, Galateau F, Ainoun $\mathrm{F}$ et al. CD95 ligand-dependant endothelial cell death initiates oral mucosa damage in a murine model of acute graft versus host disease. Lab Invest 2007; 87: 417-429.

7. Murata H, Janin A, Leboeuf C, Soulier J, Gluckman E, Meignin V et al. Donor-derived cells and human graft-versus-host disease of the skin. Blood 2007; 109: 2663-2665.

8. Cailhier JF, Sirois I, Laplante P, Lepage S, Raymond MA, Brassard N et al. Caspase-3 activation triggers extracellular cathepsin $L$ release and endorepellin proteolysis. J Bio Chem 2008; 283: 27220-27229.

9. Ishii Y, Sawada T, Kubota K, Fuchinoue S, Teraoka S, Shimizu A. Injury and progressive loss of peritubular capillaries in the development of chronic allograft nephropathy. Kidney Int 2005; 67: 321-332.

10. Truman LA, Ford CA, Pasikowska M, Pound JD, Wilkinson SJ, Dumitriu IE et al. CX3CL1/ fractalkine is released from apoptotic lymphocytes to stimulate macrophage chemotaxis. Blood 2008; 112: 5026-5036; E-pub 2008 Sep 17.

11. Lauber K, Bohn E, Krober SM, Xiao YJ, Blumenthal SG, Lindemann RK et al. Apoptotic cells induce migration of phagocytes via caspase-3-mediated release of a lipid attraction signal. Cell 2003; 113: 717-730

12. Holmes A, Abraham DJ, Chen Y, Denton C, Shi-wen X, Black CM et al. Constitutive connective tissue growth factor expression in scleroderma fibroblasts is dependent on Sp1. J Biol Chem 2003; 278: 41728-41733.

13. Shi-Wen X, Leask A, Abraham D. Regulation and function of connective tissue growth factor/CCN2 in tissue repair, scarring and fibrosis. Cytokine Growth Factor Rev 2008; 19 133-144

14. Frazier K, Williams S, Kothapalli D, Klapper H, Grotendorst GR. Stimulation of fibroblast cell growth, matrix production, and granulation tissue formation by connective tissue growth factor. J Invest Dermatol 1996; 107: 404-411.

15. Laplante P, Raymond MA, Gagnon G, Vigneault N, Sasseville AMJ, Langelier Y et al. Novel fibrogenic pathways are activated in response to endothelial apoptosis: implications in the pathophysiology of systemic sclerosis. J Immunol 2005; 174: 5740-5749.

16. Laplante P, Raymond MA, Labelle A, Abe J, lozzo RV, Hebert MJ. Perlecan proteolysis induces an alpha2beta1 integrin- and Src family kinase-dependent anti-apoptotic pathway in fibroblasts in the absence of focal adhesion kinase activation. J Biol Chem 2006; 281 30383-30392.

17. Yamamoto T, Takagawa S, Katayama I, Yamazaki K, Hamazaki Y, Shinkai H et al. Animal model of sclerotic skin. I: Local injections of bleomycin induce sclerotic skin mimicking scleroderma. J Invest Dermatol 1999; 112: 456-462.
18. Mishra R, Zhu L, Eckert RL, Simonson MS. TGF-beta-regulated collagen type accumulation: role of Src-based signals. Am J Physiol Cell Physiol 2007; 292: C1361-C1369.

19. Vepachedu R, Gorska MM, Singhania N, Cosgrove GP, Brown KK, Alam R. Unc119 regulates myofibroblast differentiation through the activation of Fyn and the p38 MAPK pathway. J Immunol 2007; 179: 682-690.

20. Thomas SM, Brugge JS. Cellular functions regulated by Src family kinases. Annu Rev Cell Dev Biol 1997; 13: 513-609.

21. Tang H, Zhao ZJ, Landon EJ, Inagami T. Regulation of calcium-sensitive tyrosine kinase Pyk2 by angiotensin II in endothelial cells. Roles of Yes tyrosine kinase and tyrosine phosphatase SHP-2. J Biol Chem 2000; 275: 8389-8396.

22. Wu SS, Jacamo RO, Vong SK, Rozengurt E. Differential regulation of Pyk2 phosphorylation at Tyr-402 and Tyr-580 in intestinal epithelial cells: roles of calcium, Src, Rho kinase, and the cytoskeleton. Cell Signal 2006; 18: 1932-1940.

23. Pshezhetsky AV. Proteomic analysis of vascular smooth muscle cells treated with ouabain. Methods Mol Biol 2007; 357: 253-269.

24. Pshezhetsky AV, Fedjaev M, Ashmarina L, Mazur A, Budman L, Sinnett D et al. Subcellular proteomics of cell differentiation: quantitative analysis of the plasma membrane proteome of Caco-2 cells. Proteomics 2007; 7: 2201-2215

25. Cailhier JF, Laplante P, Hebert MJ. Endothelial apoptosis and chronic transplant vasculopathy: recent results, novel mechanisms. Am J Transplant 2006; 6: 247-253.

26. Chujo S, Shirasaki F, Kawara S, Inagaki Y, Kinbara T, Inaoki M et al. Connective tissue growth factor causes persistent proalpha2(I) collagen gene expression induced by transforming growth factor-beta in a mouse fibrosis model. J Cell Physiol 2005; 203: $447-456$.

27. Leask A, Abraham DJ. The role of connective tissue growth factor, a multifunctional matricellular protein, in fibroblast biology. Biochem Cell Biol 2003; 81: 355-363.

28. Grotendorst GR, Duncan MR. Individual domains of connective tissue growth factor regulate fibroblast proliferation and myofibroblast differentiation. Faseb J 2005; 19 : 729-738.

29. Abreu JG, Ketpura NI, Reversade B, De Robertis EM. Connective-tissue growth factor (CTGF) modulates cell signalling by BMP and TGF-beta. Nat Cell Biol 2002; 4: 599-604.

30. Sasaki H, Nagura K, Ishino M, Tobioka H, Kotani K, Sasaki T. Cloning and characterization of cell adhesion kinase beta, a novel protein-tyrosine kinase of the focal adhesion kinase subfamily. J Biol Chem 1995; 270: 21206-21219.

31. Leask A. Transcriptional profiling of the scleroderma fibroblast reveals a potential role for connective tissue growth factor (CTGF) in pathological fibrosis. Keio J Med 2004; 53: 74-77.

32. Sato S, Nagaoka T, Hasegawa M, Tamatani T, Nakanishi T, Takigawa M et al. Serum levels of connective tissue growth factor are elevated in patients with systemic sclerosis: association with extent of skin sclerosis and severity of pulmonary fibrosis. J Rheumatol 2000; 27: 149-154

33. Shi-wen X, Pennington D, Holmes A, Leask A, Bradham D, Beauchamp JR et al. Autocrine overexpression of CTGF maintains fibrosis: RDA analysis of fibrosis genes in systemic sclerosis. Exp Cell Res 2000; 259: 213-224.

34. Stratton R, Shiwen X, Martini G, Holmes A, Leask A, Haberberger T et al. lloprost suppresses connective tissue growth factor production in fibroblasts and in the skin of scleroderma patients. J Clin Invest 2001; 108: 241-250.

35. Fonseca C, Lindahl GE, Ponticos M, Sestini P, Renzoni EA, Holmes AM et al. A polymorphism in the CTGF promoter region associated with systemic sclerosis. N Engl J Med 2007; 357: 1210-1220.

36. Cheng O, Thuillier R, Sampson E, Schultz G, Ruiz P, Zhang X et al. Connective tissue growth factor is a biomarker and mediator of kidney allograft fibrosis. Am J Transplant 2006; 6: 2292-2306.

37. Csencsits K, Wood SC, Lu G, Faust SM, Brigstock D, Eichwald EJ et al. Transforming growth factor beta-induced connective tissue growth factor and chronic allograft rejection. Am J Transplant 2006; 6: 959-966.

38. Luo GH, Lu YP, Song J, Yang L, Shi YJ, Li YP. Inhibition of connective tissue growth factor by small interfering RNA prevents renal fibrosis in rats undergoing chronic allograft nephropathy. Transplant Proc 2008; 40: 2365-2369.

39. Thomas SM, Soriano P, Imamoto A. Specific and redundant roles of Src and Fyn in organizing the cytoskeleton. Nature 1995; 376: 267-271.

40. Shi W, Haberland ME, Jien ML, Shih DM, Lusis AJ. Endothelial responses to oxidized lipoproteins determine genetic susceptibility to atherosclerosis in mice. Circulation 2000; 102: $75-81$.

Supplementary Information accompanies the paper on Cell Death and Differentiation website (http://www.nature.com/cdd) 